\title{
The Cognitive Ontogenesis of Predicate Logic
}

\author{
Pieter A. M. Seuren
}

\begin{abstract}
Since Aristotle and the Stoa, there has been a clash, worsened by modern predicate logic, between logically defined operator meanings and natural intuitions. Pragmatics has tried to neutralize the clash by an appeal to the Gricean conversational maxims. The present study argues that the pragmatic attempt has been unsuccessful. The "softness" of the Gricean explanation fails to do justice to the robustness of the intuitions concerned, leaving the relation between the principles evoked and the observed facts opaque. Moreover, there are cases where the Gricean maxims fail to apply. A more adequate solution consists in the devising of a sound natural logic, part of the innate cognitive equipment of mankind. This account has proved successful in conjunction with a postulated cognitive mechanism in virtue of which the universe of discourse (Un) is stepwise and recursively restricted, so that the negation selects different complements according to the degree of restrictedness of Un. This mechanism explains not only the discrepancies between natural logical intuitions and known logical systems; it also accounts for certain systematic lexicalization gaps in the languages of the world. Finally, it is shown how stepwise restriction of Un produces the ontogenesis of natural predicate logic, while at the same time resolving the intuitive clashes with established logical systems that the Gricean maxims sought to explain.
\end{abstract}

\section{Introduction}

The clash between natural logical intuitions and formal logic has beset the discipline of logic ever since its Aristotelian beginnings. Even nowadays the relation between language, logic, and cognition is still utterly unclear. Modern logicians have striven to make logic noncognitive and mathematical, thereby blocking further insight into the cognitive basis of logic. The rich tradition in predicate logic, developed from Aristotle on all the way through the Middle Ages till the twentieth century-a tradition in which logic, language, and cognition were intimately intertwined-was sum-

Received October 7, 2012; accepted January 7, 2013

2010 Mathematics Subject Classification: Primary 00-22; Secondary 03A99

Keywords: Abelard, Aristotle, Boethius, Hamilton, natural logic, ontogenesis of logic, Russell, undue existential import

(C) 2014 by University of Notre Dame 10.1215/00294527-2798718 
marily dismissed, mainly by Bertrand Russell, on the facile grounds that this logic was faulty. That, in the process, the baby was thrown out with the bathwater was not recognized. The only connection modern logicians are prepared to accept is in the area of syllogistic reasoning. But that is precisely what the present study is not about, since reasoning, though no doubt a cognitive faculty, tells us little about the structural and semantic properties of natural language.

Then, as far as linguistics is concerned, the "cognitivist" linguists and the pragmaticists attempt to bridge the gap between natural logical intuitions and formal logic by the introduction of the Gricean conversational maxims, regarded as the codification of principles of rational linguistic interaction. Generally, cognitivist linguists and pragmaticists decline to consider any possible relevance of logic for the study of language, which is a pity because the system of natural logic together with one or two cognitive principles provides a much stronger basis for an explanation of the discrepancies between logical systems and natural logical intuitions than the Gricean maxims will ever be able to afford. ${ }^{1}$ Chomskyan linguists are more open to logic in that they accept a notion of "logical form" (LF), but the relation of LF with actual logic has remained totally obscure. In cognitive psychology and psycholinguistics, there is a strong tendency to avoid logic as an explanatory factor. The focus nowadays is mostly on experiments meant to elucidate processing mechanisms, in particular the relation between cognitive and neurological structures and processes, to the detriment, unfortunately, of more theoretical concerns.

Montague's combination of grammar and semantics comes closest. It uses logic, more precisely logical model theory, as a tool in its quest for a semantics of natural language, in that sentence meanings are seen as functions selecting sets of possible worlds. But this application of logic has failed to convince (see Seuren [20, Chapter 9]), mainly because (a) it has no bearing on the cognitive reality of natural language, (b) it has no answer to the "propositional attitudes" problem, (c) it fails to account for scope differences, and (d) it aims at formalizing bottom-up parsing, whereas linguistic competence is defined by top-down production (see Seuren [17], [19]).

Meanwhile, the clash between natural logical intuitions and established logical systems has remained unsolved. It is argued here that the solution lies in the natural, species-specific implicit logic that develops spontaneously, according to an innate program, in each human individual. The present study is an attempt at reconstructing this logic by hypothesis. This is an empirical enterprise kept in check by the empirical observation of natural logical intuitions and of more directly linguistic facts - a common method in the human sciences.

This research program implies that the study of logic should be reunited with that of language and cognition. To this end, it is necessary to re-examine the foundations of logic. It then becomes clear that the neglect of traditional logic on the part of contemporary standard predicate logic has led to a substantial impoverishment of professionally sustained views of logic. The old square of opposition and Hamilton's much-despised triad of contraries turn out to be subparts of a generalized system of natural logic, the hexagon of logical relations (see Blanché [1], [2]), which is much richer and more functional than standard modern logic. The fault of undue existential import (UEI) is easily removed, in terms of strict bivalence, by declaring A-type sentences false when the restrictor class is null, though an empirically more 
adequate answer to UEI is the introduction of a third truth value of radical falsity for cases where UEI plays up.

To explain certain systematic facts of nonlexicalization across languages and to account for natural logical intuitions, the polarity division hypothesis is proposed, which, by a process of stepwise restriction of the universe of discourse and thus of the complement selected by negation, provides an adequate explanation for the facts at issue in terms of the natural logic developed. This makes the Gricean maxims otiose.

\section{What is (a) Logic?}

In defining what logic, or a logic, is, we must inevitably bring up many elementary notions that are familiar to any beginning logician and should, therefore, not figure in an article in a professional logical journal. Yet many other, equally basic, notions must be brought up that may be familiar to professional logicians but are not or hardly explained in elementary logic textbooks and are thus not or hardly known to wider circles of logic aficionados, such as the metalogical relations of entailment, contrariety, subcontrariety, equivalence, contradiction, and logical independence. Since these latter notions are essential to the analyses and arguments proposed below, there is no other way than to go through the lot, whether well known or not. Moreover, the perspective from which the basics of logic are looked at in the present study is so different from what is found in logical circles today that an explicit treatment is called for. Professionals will, therefore, have to bear with me.

A logic, any logic, is a system that formally defines relations of entailment or contrariety among sentences. A sentence $P$ entails a sentence $Q$, or $Q$ logically follows from $P(P \vdash Q)$, just in case whenever $P$ is true, $Q$ must also be true in virtue of the meanings of $P$ and $Q$. Thus, Alex has been murdered entails Alex is dead, because it is in the meanings of (the main predicates of) these sentences that whoever has been murdered is dead. To say that someone has been murdered but is not dead is inconsistent. Clearly, when $P \vdash Q, P$ and not- $Q$ (we write $\neg Q$ ) are inconsistent or, we say, they are contraries (we write $P \times Q$ ), in that they cannot be both true at the same time (though they may be false at the same time), in virtue of their meanings. The negation not $(\neg)$ is a binary truth-value switch, changing the value from "true" to "false," and vice versa. $P$ and $Q$ are contradictories $(P * Q)$, in any given universe of discourse $\mathbf{U n}$, just in case $P$ and $Q$ cannot both be true nor both be false at the same time: the truth of the one implies the falsity of the other, either way. Given this definition of $\neg$, for any sentence $P, P$ and $\neg P$ are by definition contradictories in any well-defined universe of discourse Un in which $P$ has a truth value.

I am using the term sentence where many textbooks use the term proposition. Even though this use of the term sentence is not coextensive with the way the term is used in linguistics, I prefer sentence to proposition because a sentence is a type-level unit, whereas a proposition, taken in the sense of a hic et nunc mental act of assigning a property to a (set of) individual(s) or to an $n$-tuple of (sets of) individuals, is a token-level event. (In other publications I use the term L-proposition, instead of what I call sentence here. This term denotes the type-level cognitive input to the grammar of any specific language, but it is too theory-dependent to be used here.) Logic is 
to do with type-level relations between sentences or, rather, sentence types, not with token-level events. If two token utterances, expressing two token propositions, are, say, contraries, they are so in virtue of the fact that they instantiate type-level sentences whose contrariety is determined by their type-level meanings. Moreover, the use of the term sentence instead of proposition makes it more natural to extrapolate from logic as such to the language as a whole - a signal feature of the approach proposed here. I will, however, follow conventional terminology when referring to propositional functions, propositional logic, or propositional operators.

A logical system $\Sigma$ depends on a restricted set of words (or morphemes), its constants or operators, whose meanings allow for analytically necessary inferencesthat is, inferences valid for all possible situations. In the logics we operate with, there are only a handful of constants, mainly the words some, all, not, and, and or. There are a few more, but we will limit ourselves to these. In certain well-defined positions in sentential structures, these operators allow for analytically necessary inferences regardless of what lexical material fills the rest of the sentences involved. Thus, the operators all and some can be defined in such a way that if all flags are green is true, it follows by entailment that some flags are green is also true, and likewise for all sentences of the same types with different lexical predicates: all Romans are mortal entails some Romans are mortal, and so forth. We generalize by replacing the predicates flag or Roman by the lexical variable $R$ ("restrictor term") and the predicates green and mortal by the lexical variable $M$ ("matrix term"). And we say that the sentence type all $R$ is $M$ entails the sentence type some $R$ is $M$, given the definitions of all and some that underlie this entailment.

In general, a logical system $\Sigma$ is defined by its values on the following parameters:

(a) the axioms of $\Sigma$;

(b) the meanings of the operators (constants) of $\Sigma$;

(c) the values of the lexical variables of $\Sigma$.

Among the axioms, we normally count the principle of contradiction (no sentence token can be both true and not true at the same time) and strict bivalence (there are precisely two truth values, true and false). ${ }^{2}$ As regards (c), the values of the lexical variables, when we work with a sentence type like $A l l R$ is $M$, it should be specified that the variables $R$ and $M$ range over predicates (propositional functions), not over whole sentences, and when we work with a formula type like $A \wedge B$, it should be specified that the variables $A$ and $B$ stand for either sentences or propositional functions (though when they stand for propositional functions, the function form $M(x)$ is preferred, where $M$ is a lexical predicate). But what counts most in the present context is parameter (b), which demands a specification of the meanings of the nonvariable constants or operators, so that the metalogical relations of $\Sigma$ follow from these meanings.

The notion of logic is thus dependent on the notion of meaning: logic is to do with analytical truth, in the Kantian sense. Another way of saying the same is to say that logic defines not ordinary (synthetic) truth but validity or analytically necessary truth, or truth in all possible situations. One can, of course, cavil about the fact that there is neither unanimity nor clarity as regards the notion of linguistic meaning, but I will not do that here. Though, as a linguist, I hold that meaning is more than truth conditions, it suffices here to assume that conditions of (synthetic) truth are a central 
a.

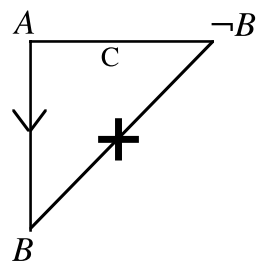

Elementary

logical

triangle b.

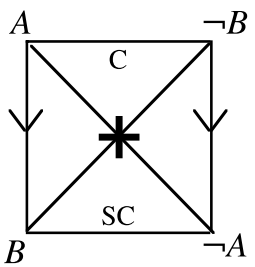

Square of

opposition c.

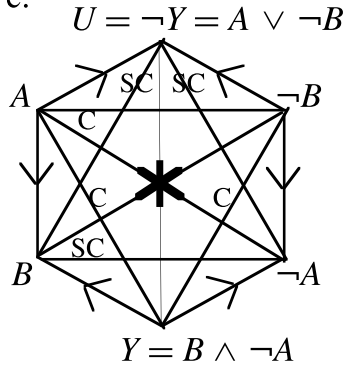

Logical

hexagon

Figure 1 The construction of the logical hexagon from entailment or contrariety.

element in the definition of meaning. Other elements of meaning are not relevant in the present context.

The following metalogical relations have so far been defined: entailment, contrariety, and contradictoriness. Further metalogical relations are logical equivalence, subcontrariety, and logical independence. Two sentences $P$ and $Q$ are logically equivalent $(P \equiv Q$ ) just in the case when $P \vdash Q$ and $Q \vdash P$. Logical equivalence is thus two-way entailment. In the following, when we speak of entailment we mean one-way entailment; for two-way entailment we use the term logical equivalence. Two sentences $P$ and $Q$ are subcontraries $(P \times Q)$ just in the case when $P$ and $Q$ cannot both be false at the same time, though they may be true at the same time. Two sentences $P$ and $Q$ are logically independent (no symbol needed) just in the case when they can at the same time be both true, both false, or the one can be true while the other is false. ${ }^{3}$

Given this metalogical toolkit, we can set up, for any given sentences $A$ and $B$, the elementary logical triangle $\langle A, B, \neg B\rangle$ of Figure 1.a. ${ }^{4}$ This triangle can be extended to a square in virtue of the fact that when $P \vdash Q, \neg Q \vdash \neg P$ (the theorem of contraposition). This is easily shown: if it is the case that whenever $P$ is true $Q$ must also be true, the truth of $\neg Q$ necessitates the truth of $\neg P$, at least in a bivalent logic with only the values true and false and nothing in between nor anything outside these two values. We can thus extend the elementary logical triangle to the square structure $\langle A, B, \neg B, \neg A\rangle$ of Figure 1.b. In this square, the new metalogical relation of subcontrariety between $B$ and $\neg A$ has arisen, as an extra bonus so to speak. That in Figure 1.b, $B$ and $\neg A$ are subcontraries is easily shown: when $B$ is false, $\neg B$ is true, and hence $\neg A$ is true; when $\neg A$ is false, $A$ is true, and hence $B$ is true.

We can go further, following Blanché [1], [2], and construct a logical hexagon, as in Figure 1.c, with the help of the operators and $(\wedge)$ and or $(\vee)$, just like $\neg$, taken from propositional logic. In the logical hexagon, $Y$ is defined as "both $B$ and $\neg A$ " ( $B \wedge \neg A)$, and $U$ as "either $A$ or $\neg B$ " $(A \vee \neg B)$. One notices that the logical hexagon combines the triad of contraries $\langle A, Y, \neg B\rangle$ with the triad of subcontraries $\langle B, \neg A, U\rangle$, while the square structure $\langle A, B, \neg B, \neg A\rangle$ is also preserved. We will see below that this fact is indispensable for an understanding of the relation between language, logic, and cognition. 
It follows from the foregoing that any relation of either entailment or contrariety automatically, in virtue of the definitions of the metalogical relations and of the meanings given for $\neg, \wedge$, and $\vee$, gives rise to a logical hexagon.

\section{Some Systems of Predicate Logic}

3.1 The square, the defect of UEI, and standard modern predicate logic The inventor, or discoverer, of logic in the Western world was Aristotle (384-322 BCE). He discovered the metalogical relation of contrariety and built up a system of predicate logic for the quantifying words all and some, with the help of the other metalogical relations of entailment and contradiction, and the propositional operator not $(\neg){ }^{5}$ Aristotle did not explicitly take into consideration the relation of subcontrariety; that notion was introduced a few centuries after his death. (As far as we know, by his commentator Apuleius, who lived in the second century CE; see Seuren [18, p. 152].) He distinguished the following eight sentence types, presented here in a new notation which distinguishes the external or sentence negation $(\neg)$ from the internal or predicate negation $(*){ }^{6}$

$\begin{array}{llll}\text { All } R \text { is } M & \text { A } & \text { All } R \text { is not } M & \text { A* }^{*} \\ \text { Some } R \text { is } M & \text { I } & \text { Some } R \text { is not } M & \mathbf{I}^{*} \\ \text { Not all } R \text { is } M & \neg \mathbf{A} & \text { Not all } R \text { is not } M & \neg \mathbf{A}^{*} \\ \text { No (= not some) } R \text { is } M & \neg \mathbf{I} & \text { No } R \text { is not } M & \neg \mathbf{I}^{*}\end{array}$

In Aristotle's logic, $\mathbf{A} \times \neg \mathbf{I}$, and hence $\mathbf{A} \vdash \mathbf{I}$, which, according to the pattern of Figure 1.b, gives rise to the square structure of Figure 2.a.

This logic, as far as it goes, is exceptionless. However, Aristotle's logical commentators, in particular the Latin author Apuleius (c. 125-180), the Greek Ammonius (c. 440-520), and the Roman Boethius (c. 480-524) thought that they could improve or streamline Aristotle's logic by positing what are now known as the conversions, that is, the equivalence of $\neg \mathbf{A}$ and $\mathbf{I}^{*}$ and of $\neg \mathbf{I}$ and $\mathbf{A}^{*}$. Generally, the conversions say that the internal negation can be substituted, salva veritate, for the external negation, provided that the universal and the existential quantifier change places (double external or internal negation cancels out). Another way of stating the conversions is to say that $\mathbf{A}$ and $\mathbf{I}$ are duals (see Löbner [12]).

Intuitively, this makes sense, since, for example, not all flags are green is felt to be equivalent with some flags are not green, and similarly for the other conversion

a.

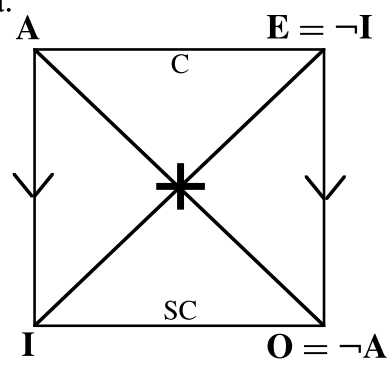

b.

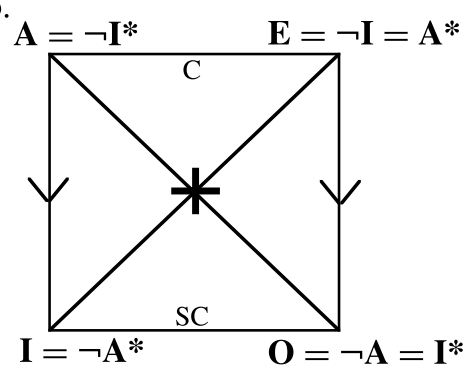

Figure 2 The classic square (a) in its pure form, (b) with the conversions. 
forms. What Aristotle's commentators failed to see was that by introducing the conversions into the system, which Aristotle had not done, they restricted the validity of the system to the situations where the $R$-class is nonnull, or $\llbracket R \rrbracket \neq \emptyset .^{7}$ I therefore distinguish between Aristotle's own logic, later completed and reformulated by the French medieval philosopher Pierre Abélard or Petrus Abaelardus (1079-1142), known in the English-speaking world as Peter Abelard, which I call AristotelianAbelardian predicate logic (AAPL) on the one hand, and Aristotelian-Boethian predicate logic (ABPL) on the other. Both are square structures, the former without the conversions (see Figure 2.a) and the latter with the conversions (see Figure 2.b). The latter is famously known as the traditional square of opposition (henceforth the square), which dominated predicate logic for about 1500 years, until the early 1900s. ${ }^{8}$

When $\llbracket R \rrbracket=\emptyset$, the square, with the conversions, becomes inconsistent. Abelard observed that if $\neg \mathbf{A}$ is taken to be equivalent with $\mathbf{I}^{*}$, then $\mathbf{I}$ and $\mathbf{I}^{*}$ are not subcontraries, because both $\mathbf{I}$ and $\mathbf{I}^{*}$ are false when $\llbracket R \rrbracket=\emptyset$ : some Romans are mortal and some Romans are not mortal are both false when there are no Romans, and so are all Romans are mortal and some Romans are not mortal (see [18, p. 176]). An alternative way of showing the same is this: if, in cases where $\llbracket R \rrbracket=\emptyset$, all Romans are mortal is considered true, then, in the square, some Romans are mortal must also be true, since $\mathbf{A} \vdash \mathbf{I}$ (the "subaltern" entailment schema). But some Romans are mortal requires, in virtue of the meaning of some, that there be Romans, quod non. If all Romans are mortal cannot be true when $\llbracket R \rrbracket=\emptyset$, then it must be false (in virtue of the axiom of strict bivalence). But if all Romans are mortal is considered false while $\llbracket R \rrbracket=\emptyset$, some Romans are not mortal, now considered equivalent with not all Romans are mortal on account of the conversions, must be true. But this again requires that there be Romans, again quod non. Therefore, all Romans are mortal can neither be true nor be false when $\llbracket R \rrbracket=\emptyset$, which makes ABPL faulty. The term commonly used for this logical defect is undue existential import (UEI). While AAPL is logically faultless, ABPL is faulty because it suffers from UEI.

To make the square sound (under the axiom of strict bivalence), it is necessary either to eliminate the conversions, which are then, as is shown below, replaced with the one-way entailments $\mathbf{A} \vdash \neg \mathbf{I}^{*}$ and $\mathbf{I} \vdash \neg \mathbf{A}^{*}$, or to keep the conversions, which practically means eliminating the square structure. The former solution considers A-type sentences false when $\llbracket R \rrbracket=\emptyset$. It was adopted by Abelard (and implicitly by Aristotle himself), reflected in AAPL. The latter solution considers A-type sentences true when $\llbracket R \rrbracket=\emptyset$. It was adopted by modern logic, under the guidance of, in particular Gottlob Frege (1848-1925) and Bertrand Russell (1872-1970), and became the standard doctrine during the 20th century, defining standard modern predicate logic (SMPL). Both are valid logical systems. The difference depends on the definition of the quantifying word all. ${ }^{9}$

It is shown below that SMPL, which keeps the conversions but drops the subalterns, leads to such an impoverished system that one can no longer speak of a square, while AAPL, which keeps the subalterns but drops the conversions, though losing some logical power compared with ABPL (the classic square), remains a fairly powerful system.

3.2 The Hamiltonian triad of contraries The Scottish philosopher Sir William Hamilton (1788-1856) developed a system of predicate logic, the Hamiltonian triad 
a.

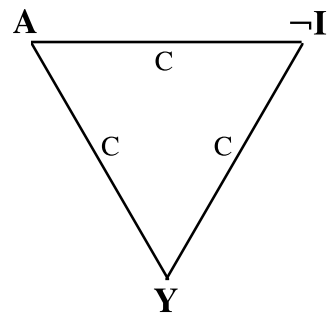

b.

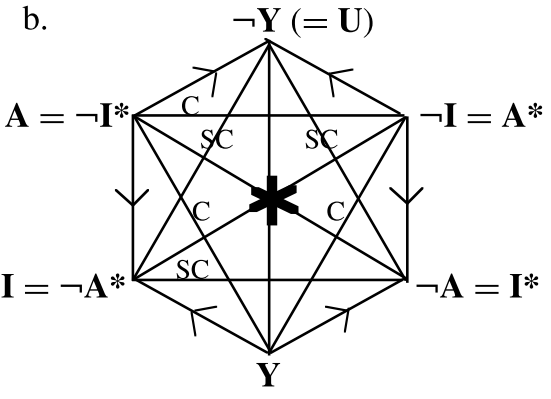

Figure 3 Hamilton's triad of contraries expanded into the Blanché hexagon.

of contraries, one element of which consists in the positing of three mutually contrary sentence types, all Romans are mortal (type A); some but not all Romans are mortal (type Y); and no Romans are mortal (type $\neg \mathbf{I}$ ) (see Hamilton [4]). ${ }^{10}$ The Hamiltonian triad is represented in Figure 3.a.

It should be noted that the Hamiltonian Y-vertex represents as a logical sentence type, characterized by a special existential operator, what in the neo-Gricean literature is taken to be a pragmatically induced scalar implicature ("some but not all"). This highlights the difference between the customary pragmatic approach and the logical approach favored here. It will become clear further down that this logical approach has an explanatory potential that far exceeds the possibilities of the pragmatic approach.

Hamilton was not worried about UEI: he simply assumed that $\llbracket R \rrbracket \neq \varnothing$. $\mathrm{He}$ did not have to worry about UEI, because UEI does not vitiate his system, which, the conversions not being part of it, is logically valid both with and without the assumption that $\llbracket R \rrbracket \neq \emptyset$. The only difference is that, when cases where $\llbracket R \rrbracket=\emptyset$ are taken into account, some logical (meta)relations are lost, whereas, when it is taken as a precondition that $\llbracket R \rrbracket \neq \emptyset$, the system is complete in the sense that not a single pair made up from the twelve possible expressions in the system is logically independent. (The twelve possible expressions are: $\mathbf{A}, \mathbf{I}$, and $\mathbf{Y}$, plus their external negations, their internal negations, and their combined external and internal negations.)

The Hamiltonian triad can be extended to the Blanché logical hexagon in the following way. The contradictory of $\mathbf{Y}, \neg \mathbf{Y}$, or, if you like, $\mathbf{U}$, is $\mathbf{A} \vee \neg \mathbf{I}$ (A and $\neg \mathbf{I}$ being the two remaining possibilities when $\mathbf{Y}$ is excluded, as shown in Figure 3.a); hence $\mathbf{A} \vdash \neg \mathbf{Y}$ and $\neg \mathbf{I} \vdash \neg \mathbf{Y}$; the contradictory of $\neg \mathbf{I}$ is $\mathbf{I}$, semantically defined as "there is at least one $x$ such that both $R(x)$ and $M(x)$ are true"; since $\mathbf{Y}$ and $\neg \mathbf{I}$ are contraries, $\neg \mathbf{Y}$ and $\mathbf{I}$ are subcontraries; likewise for the contrary pair $\mathbf{A}$ and $\mathbf{Y}$, which makes their negations $\neg \mathbf{A}$ and $\neg \mathbf{Y}$ subcontraries, and for the contrary pair $\mathbf{A}$ and $\neg \mathbf{I}$, which makes $\mathbf{I}$ and $\neg \mathbf{A}$ subcontraries. The original Hamiltonian triad of contraries is thus extended with a triad of subcontraries. Moreover, given that the quantifying word all is defined as "for all $x$ such that $R(x)$ is true, $M(x)$ is also true, and there is at least one $x$ such that $R(x)$," it follows that if $\mathbf{A}$ is true, $\mathbf{I}$ (defined as "there is at least one $x$ such that both $R(x)$ and $M(x)$ are true") must also be true, which establishes the subaltern entailment $\mathbf{A} \vdash \mathbf{I}$ and hence, by contraposition, $\neg \mathbf{I} \vdash \neg \mathbf{A}$. Finally, if it is assumed that $\llbracket R \rrbracket \neq \emptyset$, the conversions hold, as stated in Figure 3.b. 
Table 1 VS-model for the semantically independent sentences A, B, and C.

\begin{tabular}{|r|c|c|c|c|c|c|c|c|}
\hline valuations (= Un): & 1 & 2 & 3 & 4 & 5 & 6 & 7 & 8 \\
\hline $\boldsymbol{A}$ & $\mathrm{T}$ & $\mathrm{F}$ & $\mathrm{T}$ & $\mathrm{F}$ & $\mathrm{T}$ & $\mathrm{F}$ & $\mathrm{T}$ & $\mathrm{F}$ \\
\hline $\boldsymbol{B}$ & $\mathrm{T}$ & $\mathrm{T}$ & $\mathrm{F}$ & $\mathrm{F}$ & $\mathrm{T}$ & $\mathrm{T}$ & $\mathrm{F}$ & $\mathrm{F}$ \\
\hline $\boldsymbol{C}$ & $\mathrm{T}$ & $\mathrm{T}$ & $\mathrm{T}$ & $\mathrm{T}$ & $\mathrm{F}$ & $\mathrm{F}$ & $\mathrm{F}$ & $\mathrm{F}$ \\
\hline
\end{tabular}

\section{Valuation Space Analysis}

It has just been said that, as long as it is assumed that $\llbracket R \rrbracket \neq \emptyset$, no two of the twelve sentence types in the Blanché hexagon are logically independent. (The same goes for its subpart, ABPL, i.e., the square). To prove that these and other similar statements are true would take a considerable amount of time and of intricate puzzling. Little wonder that such theorems as these have never been systematically investigated in the history of logic. To counter this problem (and for other reasons as well), I have developed a system, called valuation space analysis (VS-analysis), a user-friendly system that makes one see all those things at a glance. It is used a great deal in the following. Here is how it works. ${ }^{11}$

First we need to know what is meant by valuation space. To make that clear, we need to know what is meant by a valuation, and the best way to show that is by means of a simple toy example. Take three contingent (i.e., neither necessarily true nor necessarily false) and logically independent sentences, say, $A, B$, and $C$. In any given situation, these are either true or false (the only truth values we work with: strict bivalence). This gives $8\left(2^{3}\right)$ possibilities, as shown in Table 1 ("T" stands for true, "F" for false). ${ }^{12}$

Each column (from 1 to 8 ) represents a specific situation, also called valuation. Together, they form the universe of discourse (Un) for $A, B$, and $C$. Now we give the following definition of valuation space:

The valuation space (VS) /P/ of a sentence $P$ is the set of valuations (situations) in which $P$ is valued true.

Given Table 1: $/ A /=\{1,3,5,7\}$,

$$
\begin{aligned}
& / B /=\{1,2,5,6\}, \\
& / C /=\{1,2,3,4\} .
\end{aligned}
$$

Suppose that $A, B$, and $C$ are not logically independent, for example, because $A \vdash C$. In that case all valuations where $A$ is true and $C$ is false are ruled out, or are inadmissible, on analytic grounds: such situations are per se impossible. Therefore, if $A \vdash C$, the valuations 5 and 7 are inadmissible and are removed from Un.

The logical compositions follow automatically, according to the well-known truth tables. For example:

$$
\begin{array}{ll}
/ \neg A /=\{2,4,6,8\}, & / A \wedge \neg B /=\{3,7\}, \\
/ A \wedge B /=\{1,5\}, & / \neg A \vee B /=\{1,2,4,5,6,8\}, \\
/ A \vee B /=\{1,2,3,5,6,7\}, & / \neg(A \wedge B) /=\{2,3,4,6,7,8\}, \quad \text { and so on. }
\end{array}
$$

It is now easily seen that the metalogical relations are expressible in terms of VSs (note that contradiction is the combination of contrariety and subcontrariety): 


$\begin{array}{lll}\text { Entailment: } & P \vdash Q & \text { if and only if } / P / \subseteq / Q / ; \\ \text { Equivalence: } & P \equiv Q & \text { if and only if } / P /=/ Q / ; \\ \text { Contrariety: } & P \times Q & \text { if and only if } / P / \cap / Q /=\emptyset ; \\ \text { Subcontrariety: } & P \times Q & \text { if and only if } / P / \cup / Q /=\mathbf{U n} ; \\ \text { Contradiction: } & P \ngtr Q & \text { if and only if } / P / \cap / Q /=\emptyset \text { and } \\ & & / P / \cup / Q /=\mathbf{U n} ; \\ \text { Logical independence: } & \text { if and only if } / P / \cap / Q / \neq \emptyset \neq P \neq Q \text { and } \\ & & / P / \cup / Q / \neq \mathbf{U n} .\end{array}$

At this point we need to be more specific as regards the internal structure of the sentence types that we have so far identified as $\mathbf{A}, \mathbf{I}, \mathbf{Y}$, and so on. To this end, we use a variety of the language of generalized quantifiers, distinguishing a grammatical category of quantifiers, which are treated as binary higher-order predicates, that is, predicates over pairs of sets (or sets of sets, etc.). The customary symbol $\forall$ (the universal quantifier) is used for "all" and $\exists$ (the inclusive existential quantifier) for "some perhaps all." For our special purpose, the symbol $\exists^{\mathrm{exc}}$ (the exclusive existential quantifier) is added for "some but not all" or "only some." Since $\forall, \exists$, and $\exists^{\text {exc }}$ are considered to be binary predicates, they have two terms, a subject term and an object term. We take the matrix predicate $M$ to form the subject term and the restrictor predicate $R$ to form the object term. ${ }^{13}$ The types $\mathbf{A}, \mathbf{A}^{*}, \mathbf{I}, \mathbf{I}^{*}, \mathbf{Y}, \mathbf{Y}^{*}$ now have the following logical form:
A: $\forall x(M x, R x)$,
$\mathbf{I}: \exists x(M x, R x)$,
$\mathbf{Y}: \exists^{\operatorname{exc}}(M x, R x)$,
$\mathbf{A}^{*}: \forall x(\neg M x, R x)$,
$\mathbf{I}^{*}: \exists x(\neg M x, R x)$,
$\mathbf{Y}^{*}: \exists^{\mathrm{exc}}(\neg M x, R x)$.

The next step is to define the satisfaction conditions (meanings) of the quantifying predicates. We do this in terms of set theory. For the purpose of the logical hexagon and the square, the three quantifiers $\forall$ ("all"), $\exists$ ("some perhaps all"), and $\exists^{\text {exc }}$ ("some but not all," or "only some") are defined as follows (the usual notation of denoting the complement of $X$ in any given domain of entities by a horizontal line above a set-denoting expression is followed here): ${ }^{14}$

$$
\begin{aligned}
\llbracket \forall \rrbracket= & \{\langle Y, X\rangle \mid X \subseteq Y \text { and } X \neq \emptyset\} \\
& \text { (the extension of the binary higher-order predicate } \forall \text { is the set of } \\
& \text { all pairs of sets }\langle Y, X\rangle \text { such that } X \text { is included in or equal to } Y, \\
& \text { and } X \neq \emptyset) ; \\
\llbracket \exists \rrbracket & \{\langle Y, X\rangle \mid Y \cap X \neq \emptyset\} \\
& \text { (the extension of the binary higher-order predicate } \exists \text { is the set of } \\
& \text { all pairs of sets }\langle Y, X\rangle \text { such that the intersection of } Y \text { and } X \text { is } \\
& \text { nonnull); } \\
& \{\langle Y, X\rangle \mid Y \cap X \neq \emptyset \text { and } Y \cap X \neq \emptyset\} \text { or } \\
& \{\langle Y, X\rangle \mid Y \cap X \neq \emptyset \neq X\} \\
& \text { (the extension of the binary higher-order predicate } \exists \text { exc is the set of } \\
& \text { all pairs of sets }\langle Y, X\rangle \text { such that the intersection of } Y \text { and } X \text { is } \\
& \text { nonnull and the intersection of } \bar{Y} \text { and } X \text { is nonnull; alternatively, } \\
& \text { the intersection of } Y \text { and } X \text { is nonnull and unequal to } X \text { ). }
\end{aligned}
$$

The various sentence types are assigned to valuation spaces according to their truth conditions. ${ }^{15}$ We distinguish four spaces (leaving out, for the moment, a fifth space for the unique situation of a null universe $(\mathbf{U n}=\emptyset))$, and we stipulate the following: 
Space 1: is reserved for all valuations (situations) in Un where $\llbracket R \rrbracket \subseteq \llbracket M \rrbracket$ and $\llbracket R \rrbracket \neq \varnothing$; that is, the set of entities denoted by the predicate $R$ is nonnull and is included in or equal to the set of entities denoted by the predicate $M$;

Space 2: contains all valuations (situations) in Un, minus space 1 , where $\llbracket M \rrbracket$ and $\llbracket R \rrbracket$ have a nonnull intersection: $\llbracket M \rrbracket \cap \llbracket R \rrbracket \neq \varnothing \neq \llbracket R \rrbracket$; space 2 thus also contains the situations where $\llbracket M \rrbracket \subset \llbracket R \rrbracket$, but not those where $\llbracket R \rrbracket \subseteq \llbracket M \rrbracket$, which belong exclusively in space 1 ;

Space 3: contains all valuations (situations) in Un where $\llbracket M \rrbracket$ and $\llbracket R \rrbracket$ have a null intersection and $\llbracket R \rrbracket$ is nonnull: $\llbracket R \rrbracket \cap \llbracket M \rrbracket=\emptyset$ and $\llbracket R \rrbracket \neq \emptyset$;

Space 4: contains all valuations (situations) in Un where $\llbracket R \rrbracket=\emptyset$.

Under these definitions, the sentence types at issue have the following VSs in Un:

$$
\begin{array}{llll}
/ \mathbf{A} /=\{1\}, & / \neg \mathbf{A} /=\{2,3,4\}, & / \mathbf{A}^{*} /=\{3\}, & / \neg \mathbf{A}^{*} /=\{1,2,4\}, \\
/ \mathbf{I}=\{1,2\}, & / \neg \mathbf{I} /=\{3,4\}, & / \mathbf{I}^{*} /=\{2,3\}, & / \neg \mathbf{I}^{*} /=\{1,4\}, \\
/ \mathbf{Y} /=\{2\}, & / \neg \mathbf{Y} /=\{1,3,4\}, & / \mathbf{Y}^{*} /=\{2\}, & / \neg \mathbf{Y}^{*} /=\{1,3,4\}, \\
/ \mathbf{U} /=\{1,3,4\}, & / \neg \mathbf{U} /=\{2\}, & / \mathbf{U}^{*} /=\{1,3,4\}, & / \neg \mathbf{U}^{*} /=\{2\} .
\end{array}
$$

Therefore, among the logical relations holding in Un (besides the contradictions brought about by the negation) are the following:

$$
\begin{array}{llll}
\mathbf{A} \vdash \mathbf{I} & \{1\} \subseteq\{1,2\}, & \mathbf{A} \times \neg \mathbf{I} & \{1\} \cap\{3,4\}=\emptyset, \\
\mathbf{A}^{*} \vdash \mathbf{I}^{*} & \{3\} \subseteq\{2,3\}, & \mathbf{A} \times \mathbf{I}^{*} & \{1\} \cap\{2,3\}=\emptyset, \\
\mathbf{A} \vdash \neg \mathbf{I}^{*} & \{1\} \subseteq\{1,4\}, & \mathbf{A} \times \mathbf{A}^{*} & \{1\} \cap\{3\}=\emptyset, \\
\mathbf{I} \vdash \neg \mathbf{A}^{*} & \{1,2\} \subseteq\{1,2,4\}, & \mathbf{I} \times \mathbf{A}^{*} & \{1,2\} \cap\{3\}=\emptyset, \\
& & \neg \mathbf{I}^{*} \times \mathbf{A}^{*} & \{1,4\} \cap\{3\}=\emptyset, \\
& \neg \mathbf{A} \times \mathbf{I} & \{2,3,4\} \cup\{1,2\}=\mathbf{U n}, \\
& \neg \mathbf{A} \times \neg \mathbf{A}^{*} & \{2,3,4\} \cup\{1,2,4\}=\mathbf{U n}, \\
& \neg \mathbf{A} \subseteq \neg \mathbf{I}^{*} & \{2,3,4\} \cup\{1,4\}=\mathbf{U n}, \\
& \neg \mathbf{A}^{*} \leq \neg \mathbf{I} & \{1,2,4\} \cup\{3,4\}=\mathbf{U n}, \\
& \neg \mathbf{A}^{*} \leq \mathbf{I}^{*} & \{1,2,4\} \cup\{2,3\}=\mathbf{U n} .
\end{array}
$$

Moreover,

$$
\begin{aligned}
& \mathbf{Y} \equiv \mathbf{Y}^{*} \text { because } / \mathbf{Y} /=/ \mathbf{Y}^{*} /=\{2\} \\
& \neg \mathbf{Y} \equiv \mathbf{U} \text { because } / \neg \mathbf{Y} /=/ \mathbf{U} /=\{1,3,4\}, \\
& \mathbf{U} \equiv \mathbf{U}^{*} \text { because } / \mathbf{U} /=/ \mathbf{U}^{*} /=\{1,3,4\} .
\end{aligned}
$$

This system is consistent and free from UEI, but it does not have the conversions since $/ \mathbf{A} /=\{1\} \neq / \neg \mathbf{I}^{*} /=\{1,4\}$, and $/ \mathbf{I} /=\{1,2\} \neq \neg \mathbf{A}^{*} /=\{1,2,4\}$. Without the $\mathbf{Y}$ - and $\mathbf{U}$-vertices, it is the system originally conceived by Aristotle, until it was streamlined into the logical square by later logicians, in particular Boethius, and reconstructed by Abelard in the twelfth century (see note 8). In this system, AAPL, A, and I are no longer duals (the conversions do not hold), as we have seen. All that remains from duality are the one-way entailments $\mathbf{A} \vdash \neg \mathbf{I}^{*}$, but not vice versa, and $\mathbf{I} \vdash \neg \mathbf{A}^{*}$, but not vice versa.

This analysis allows us to draw diagrams that show up immediately the metalogical relations holding between sentence types. We have two kinds of diagram, VS-analyses ("ontological" diagrams) and polygonal representations of the logic concerned (like the square notation). The classic Aristotelian-Boethian square of opposition-with UEI and thus with the conversions-is represented in Figure 4.a 
a.

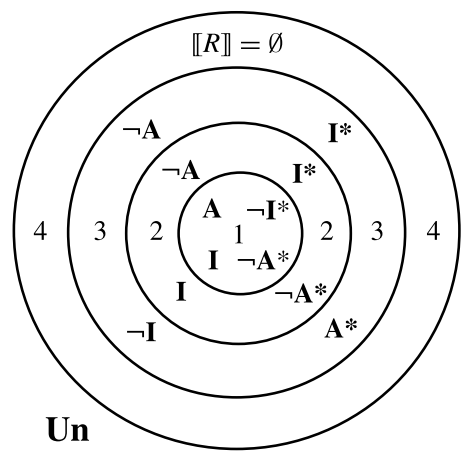

b.

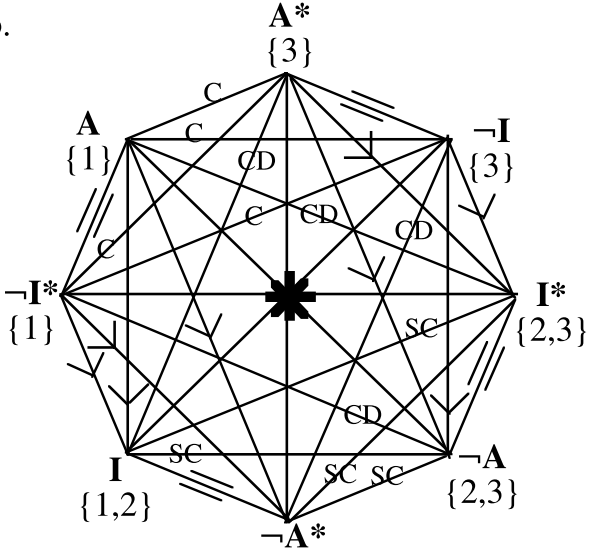

$\{1,2\}$

c.

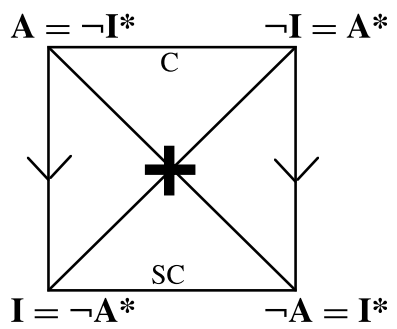

Figure 4 The VS-model, the octagon, and the square for ABPL.

as a VS-model. Note that space 4 has been left empty in Figure 4.a, because cases where $\llbracket R \rrbracket=\emptyset$ are not taken into account. That is, the system is not valid for all possible situations and thus not analytically necessary: the fault of UEI (which by itself does not mean that no inferences can be drawn, only that the inferences must be indexed for the situation classes for which they are defined). In the octagonal representation of Figure 4.b, each vertex stands for a sentence type. When every pair of logically equivalent types is amalgamated into one vertex, the square of ABPL results, as shown in Figure 4.c. ${ }^{16}$

When we do the same for AAPL (the square but without the conversions), we get Figure 5. Since Figure 5.b contains no equivalence relations, it cannot be reduced. Note that the octagon of Figure 5.b is "poorer" than that of Figure 4.b, which is complete in that each pair of vertices is connected by some (meta)relation other than logical independence.

Now we repeat this operation for standard modern predicate logic (SMPL), often called Russellian logic. The only difference with AAPL is that, in AAPL, the universal quantifier $\forall$ is defined with the condition that $\llbracket R \rrbracket \neq \emptyset$ so that $\mathbf{A}$-type and $\mathbf{A}^{*}$-type sentences are considered false in space 4 , whereas in SMPL $\forall$ is defined without that condition, so that $\mathbf{A}$ and $\mathbf{A}^{*}$ are considered true-very much against natural intuitions. This small difference has massive consequences for the logic, as one can see from Figure 6. The only (meta)logical relations that have been left are 
a.

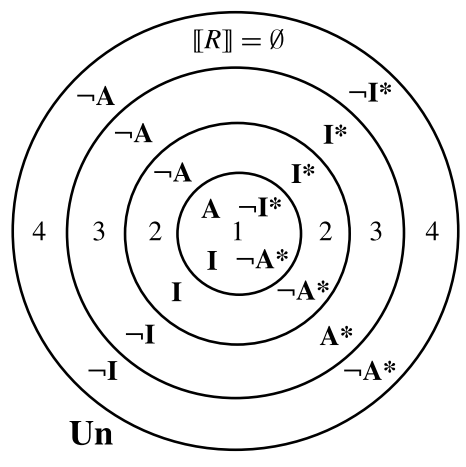

b.

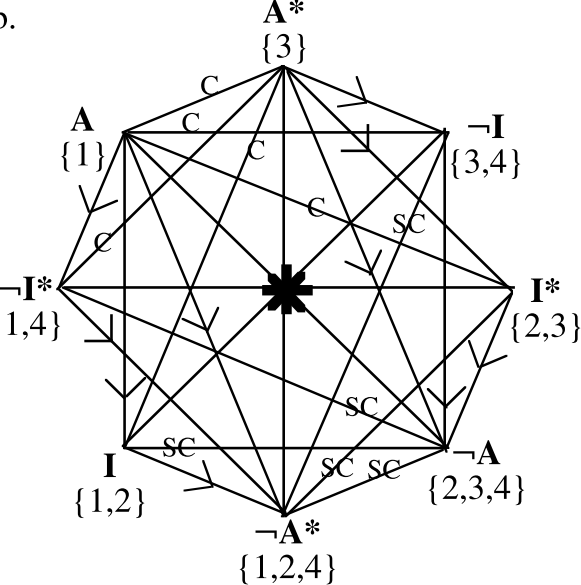

Figure 5 The VS-model and the octagon for AAPL.

a.

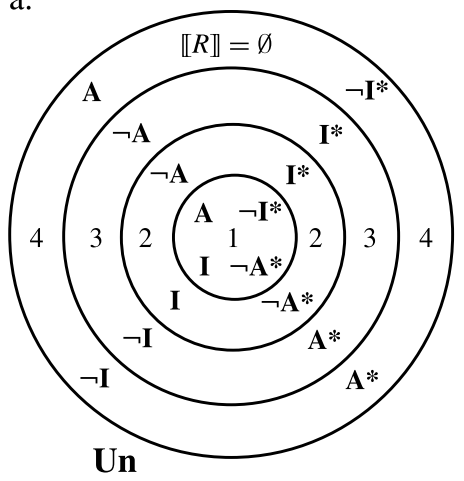

c.
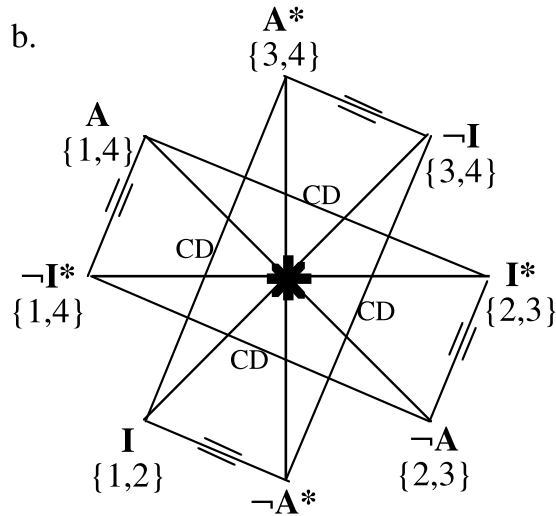

$\{1,2\}$

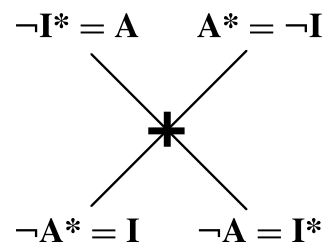

Figure 6 The VS-model and what remains of the octagon and the square for SMPL.

contradictoriness and equivalence, with the result that there is massive logical independence. The square has completely disappeared. This dramatic impoverishment has led to the disturbing fact that current textbooks of logic no longer make mention of the logical metarelations discussed above, with the exception of contradictoriness and equivalence, even though they are the backbone of any logical theory. 


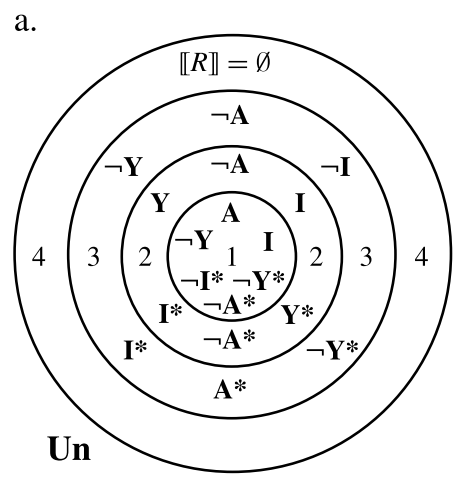

c.
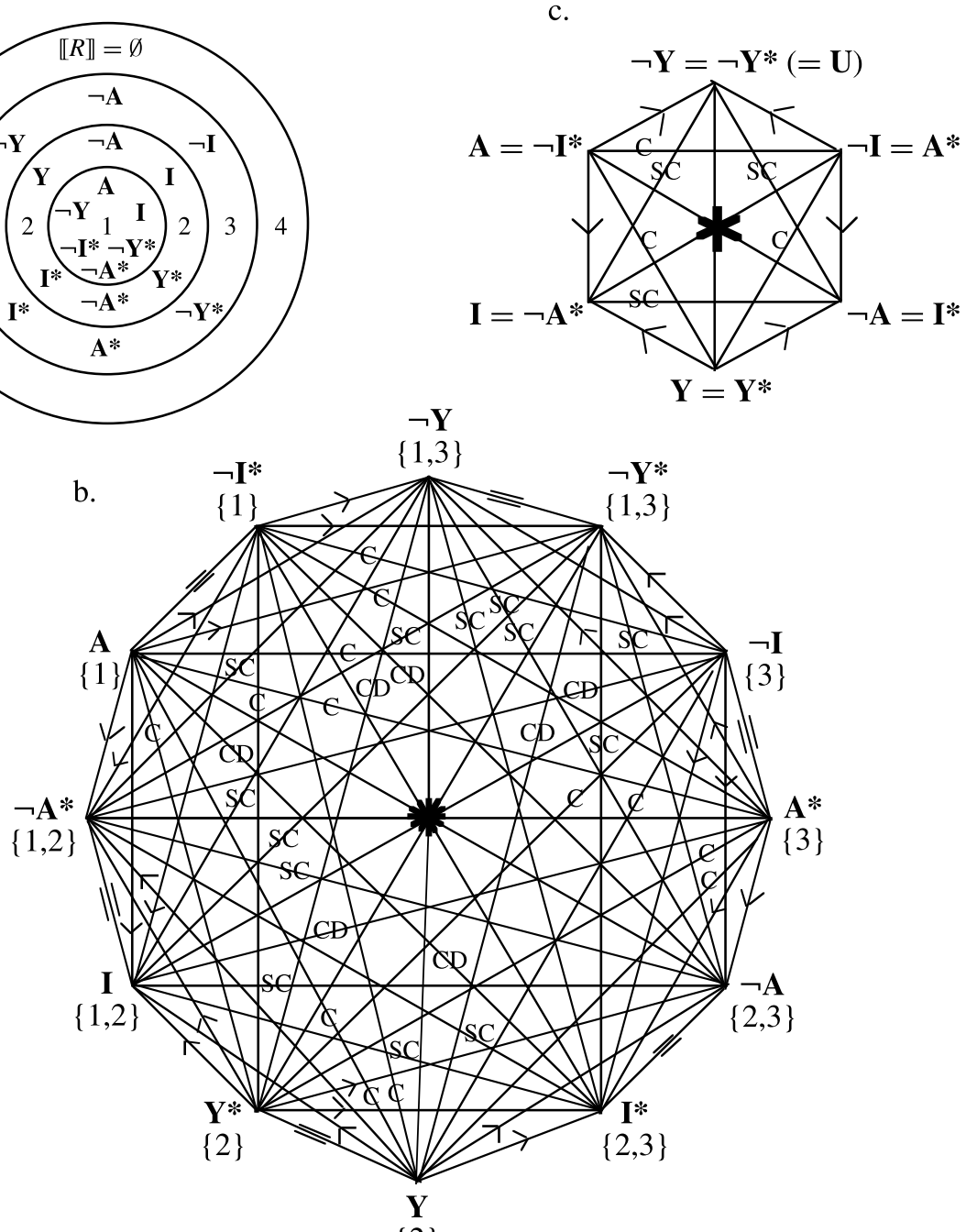

$\{2\}$

Figure 7 The VS-model, the dodecagon, and the hexagon for RGNPL.

We now do the same for the Hamiltonian triad of contraries and its extension, the Blanché logical hexagon, shown in Figure 3.b above. Here, we have twelve sentence types: $\mathbf{A}, \mathbf{Y}, \neg \mathbf{I}$, plus their external negations, their internal negations, and their external plus internal negations. The logical language used is the same as above; so are the semantic definitions of the quantifiers $\forall, \exists$, and $\exists^{\text {exc }}$. Space 4 is left out of account, so that the system has existential import and the conversions hold.

Figure 7.b looks forbidding, yet it is merely the dodecagon resulting from Figure 7.a. It contains six equivalences (all on the outer edges). When these are amalgamated into a single vertex, the result is the hexagon of Figure 7.c, which is identical to that of Figure 3.b. The Blanché hexagon is thus derivable both from the clas- 


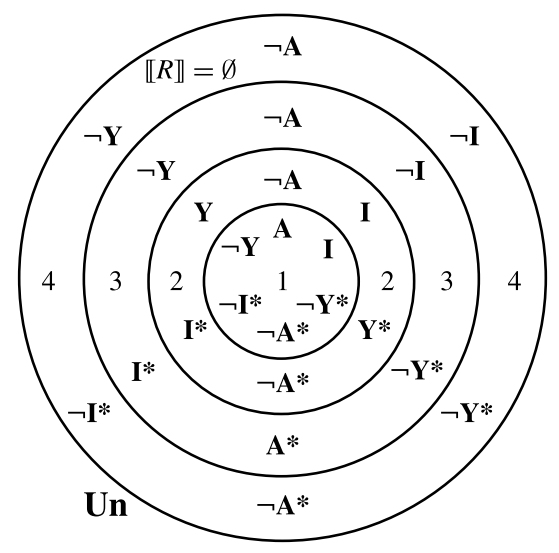

Figure 8 The VS-model for UGNPL, where $\llbracket R \rrbracket=\emptyset$ is taken into account.

sic square and from the Hamiltonian triad. In fact, the Boethian square and the Hamiltonian triad are both part of an overarching system, represented by the Blanché hexagon, for cases where $\llbracket R \rrbracket \neq \emptyset$. They differ only in that each system has made its own selection from the six available vertices: $\mathbf{A}, \mathbf{I}, \neg \mathbf{I}, \neg \mathbf{A}$ for the square and $\mathbf{A}, \mathbf{Y}$, $\neg \mathbf{I}$ for the Hamiltonian triad.

The logic corresponding to the Blanché hexagon, which encompasses the square and the Hamiltonian triad, is henceforth called generalized natural predicate logic (GNPL). Like the square, it has two forms, one without and one with existential import, depending, respectively, on whether space 4 is or is not taken into account. The variety with existential import-that is, without space 4-I call restricted generalized natural predicate logic (RGNPL). The variety without existential import-that is, with space 4 operative-I call unrestricted generalized natural predicate logic (UGNPL).

When space 4 is taken into account, the semantic definitions given for $\forall, \exists$, and $\exists^{\text {exc }}$ make $\mathbf{A}, \mathbf{A}^{*}, \mathbf{I}, \mathbf{I}^{*}, \mathbf{Y}$, and $\mathbf{Y}^{*}$ false (see Figure 8). This means that most of the equivalences of Figure $7 . b$ are gone. Only the equivalent pairs $\mathbf{Y}$ and $\mathbf{Y}^{*}$, and thus $\neg \mathbf{Y}$ and $\neg \mathbf{Y}^{*}$, remain intact. With $\llbracket R \rrbracket=\varnothing$ taken into account, the dodecagon of Figure 7.b is thus reducible to a decagon, which, however, is not presented here as a separate figure.

It is clear from the above that ABPL (the traditional square), AAPL, the Hamiltonian triad, and the Blanché hexagon all belong to the same family of logics, which we have called generalized natural predicate logic or GNPL. All logics belonging to GNPL have the universal quantifier $\forall$ defined in such a way that the $\mathbf{A}$ and $\mathbf{A}^{*}$ sentence types are false when $\llbracket R \rrbracket=\emptyset$, which conforms to natural intuition. SMPL does not belong to this family, as SMPL takes the $\mathbf{A}$ and $\mathbf{A} *$ sentence types to be true when $\llbracket R \rrbracket=\emptyset$. It is my contention that the natural logic of mankind is to be sought within RGNPL, cases where $\llbracket R \rrbracket=\emptyset$ (and other kinds of presupposition failure) being accounted for by a trivalent presuppositional logic serving as a protective envelope around the bivalent default cases (see [18, Chapter 10] and Section 5.2 below). 
What all this shows is that the square, the Hamiltonian triad, the Blanché hexagon, and in general all logics falling under UGNPL are sound systems of logic, where UEI does not rear its head. UEI begins to play a role the moment equivalences are stated. The square is sound without the equivalences known as the conversions. The same goes for the other logics belonging to the GNPL family. The addition of space 4 destroys most equivalences and thus makes the logics at issue poorer, but the systems as such, with all their logical metarelations between pairs of sentence types, are sound. The interesting, and most relevant, point is that maximal power for a logical system is attained when space 4 is not taken into account-which is precisely what natural language and natural logic do: nature has found a way to maximize logical efficiency by confining bivalence to cases where $\llbracket R \rrbracket \neq \emptyset$, no doubt for good functional reasons, reflected in the ontogenesis of predicate logic, as argued below.

A further interesting point, more directly relevant to logic as a discipline, is the following. It was said above that any logical system is defined by its axioms, the ranges of its variables, and the semantic definitions of its operators. When we take the traditional Aristotelian-Boethian square of opposition, which is a restricted system of the GNPL family, we see that it is not possible to define the universal quantifier $\forall$ in the set-theoretic manner presented above. With the condition that $\llbracket R \rrbracket \neq \emptyset$, the result is AAPL; without that condition the result is SMPL. The traditional square, with its conversions, cannot be defined in such a way that it follows from the semantic definitions of its quantifiers: the conversions have to be added as an extraneous condition, which violates the charter for a good logical system. Nevertheless, this highly understandable error has given high profile to the fact that natural logic operates within the restricting condition that $\llbracket R \rrbracket \neq \varnothing$.

\section{How Cognition Plays Its Part in Natural Logic}

5.1 From not all to some but not all We have come some way toward accounting for the discrepancies between standard predicate logic and natural logical intuitions, yet we are not quite there. Some light has been shed on the ambiguity of natural language some, which means both "some perhaps all" (the I-vertex) and "some but not all" (the Y-vertex), in that the latter is made to represent a basic-natural operator forming part of the Hamiltonian triad of contraries, while the former can be retrieved through more sophisticated reflection. The natural language universal quantifier all has been restored to a status of logical respect in that it has been shown to fit into a family of faultless and efficient logical systems, the family of RGNPL. The conversions, which would be lost to the RGNPL family under strict bivalence, have been saved by relegating cases where $\llbracket R \rrbracket=\emptyset$ to a nondefault area of predicate logic requiring a marked discourse-correcting, radical negation to produce truth (see note 9 and below), a trivalent presuppositional solution elaborated and presented in [18] and earlier publications by the same author. Yet, so far, we have been unable to account for the intuition that (1) and (2) are felt to be equivalent.

(1) Not all flags are green.

(2) Some flags are green, and some flags are not green.

In none of the logical systems discussed are they equivalent, nor does it look as if any logical system with the standard bivalent negation $\neg$ will be able to make them equivalent. 
At this stage of our investigation, the nonequivalence of (1) and (2) in all known logical systems is thus our main stumbling block, which may make it look as if the pragmatic account has an edge over the logical account proposed here. The standard pragmatic argument used to be that this equivalence follows from the Gricean first maxim of quantity (see Grice [3, p. 45]), which says, "Make your contribution as informative as is required." This, however, makes the application of this maxim dependent on ad hoc situational features, whereas the intuitive equivalence of (1) and (2) is, though situation-dependent in a logical sense, independent of ad hoc features in any given situation. Levinson [11, p. 35] cuts out the situational variability and translates the maxim as his "Q-principle": "What isn't said, isn't." This principle, however, quickly leads to absurdity. Consider a sentence like Kevin left yesterday. According to the Q-principle, this should pragmatically implicate "Kevin did not leave yesterday morning," as yesterday morning is more informative (more specific and thus stronger) than yesterday. But by the same token, Kevin left yesterday should pragmatically implicate "Kevin did not leave yesterday afternoon," "Kevin did not leave yesterday evening," and "Kevin did not leave last night." This would amount to an overall pragmatic implicature from Kevin left yesterday to "Kevin did not leave yesterday," which is absurd. The pragmatic literature is unclear on this score, but both Grice's first maxim, "Make your contribution as informative as is required," and Levinson's rendering of it in the form of his Q-principle seem untenable.

Grice's first maxim was shored up by associating it with scalar implicatures in the sense that, given a sentence $\ldots X \ldots$, where $X$ has a certain position on an implicational scale of increasing strength or restrictedness, the negation of $\ldots X \ldots$ is taken to conversationally implicate the negation of all sentences $\ldots Y \ldots$ where $Y$ is stronger than (entails) $X$. This means that Levinson's pragmatic Q-principle, "What isn't said, isn't," should be restricted to "What isn't said by means of a stronger or entailing expression, isn't." But this modification again hits the wall of absurdity. If Kevin ate some of the food is taken to pragmatically implicate "Kevin did not eat all of the food," because the speaker would have used the stronger expression all the food if she or he could have vouched for that, then the implicatures "Kevin did not eat most/half/much/a little/... of the food" should likewise be generated by this sentence, so that Kevin ate some of the food should pragmatically implicate "Kevin ate none of the food,"

Scalar orderings of predicates were developed before Grice by Jespersen [10, pp. 85-86] and Blanché [1, pp. 122-26], [2, pp. 110-19], likewise with the purpose of accounting for the restricted meaning of the negation over scalar expressions. According to Jespersen, there is a cognitive scale from "none" via "some but not all" to "all." The negation of all would then "cut off" the head of the scale and leave "some but not all," or, in Jespersen's own, somewhat quaint, language "in negativing an $A$ [that is, an A-type sentence; P. A. M. Seuren] it is the absolute element of $A$ that is negatived" (see [10, p. 86]). This, however, will not do, as taking off the "absolute element" of all leaves one with most, so that not all would suggest ("implicate") most, which it clearly does not.

Moreover, Jespersen's reasoning requires that other semantic scales should generate corresponding "implicatures," which does not seem to be the case. Consider, for example, in the realm of colors, the scale from black via grey to white, or, in the realm of temperature, from cold via warm to hot, or, in the realm of water occurring in nature, from fresh via brackish to salt, or, in the realm of light, from 
dark via dusky to bright, or, in the realm of food, from bland via savory to spicy. These do not seem to generate intuitions analogous to (1) and (2) above-certainly not with the strength of (1) and (2), where the intuition has the quality of a mutual entailment. Not white does not suggest grey, excluding black; not hot does not suggest warm, excluding cold,${ }^{17}$ nor does not salt suggest brackish, excluding fresh; not bright does not suggest dusky, excluding dark, nor does not spicy suggest savory, excluding bland. By contrast, expressions like not quite white, not quite hot, not quite salt, not quite bright, not quite spicy clearly do suggest "white/hot/salt/bright/spicy to some extent." But then, English not quite is a representative of the negated universal quantifier (German nicht ganz, French pas complètement). It does seem, therefore, that any proposed quantificational scale stands out from other possible scales.

When we look at the enormous pragmaticist literature on the subject, we must come to the sobering conclusion that the combination of the Gricean first maxim of quantity with the (in fact, Jespersonian) notion of scalar implicatures has not yielded a solution to the intuitive equivalence of (1) and (2). The discussion in Horn [5, pp. 231-45] is illuminating as regards the history of the problem, as it deals critically with a wide range of literature, some of the critique being directed at his own seminal dissertation Horn [6], but it does not provide an answer. Since no better or more specific proposal from pragmaticist quarters has seen the light, one may conclude that pragmatic approaches in general have failed to provide a valid, principled answer. Pragmatics thus has no edge over the logical approach as regards the intuitively felt equivalence of (1) and (2).

Let us, therefore, forge ahead on the so far only partially explored logical front. As long as not is taken to be a complement selector within a universe of discourse (Un) that remains constant for the logical system as a whole, as is done in all logical systems, the intuitive equivalence relation between (1) and (2) remains unexplained. ${ }^{18}$ This does not mean, however, that we are at the end of our logical Latin, since logic has so far not, or insufficiently, explored the role of Un in logical systems, in particular the consequences of progressive restriction of Un as discourse proceeds (presupposition theory) or in the course of concept formation, which is our primary concern in the present study. From a formal point of view, it is easily shown that all metalogical relations may be affected when the class of situations in which a logical system holds-its Un-is restricted from a larger to a smaller size.

We shall have a look at that in a moment. First, we wish to observe that this point of view is fruitful from a functional cognitive perspective. Humans, in their often intricate daily business, do not conduct their thinking in the terms of a philosophically and mathematically constructed, highly abstract universe of all possible situations, a universe of discourse we call $\mathbf{U n}^{\omega}$. Ordinary life efficiency requires that cognition should operate in terms of highly restricted, ad hoc "universes of discourse," where available contextual, situational, and world knowledge help to determine reference relations established by definite noun phrases (that man, the house on the corner), where words can be used that are made for specific classes of situations and thus induce presuppositions (e.g., be divorced, inducing the presupposition that the subject was married before, or come back, inducing the presupposition that the subject has gone away), and where words expressing logical concepts (all, some, etc.) can be kept within the limits imposed by such contextually and situationally restricted universes of discourse. 
To account for such Un-restriction we posit three assumptions:

(a) There is a species-specific basic-natural level of cognitive development attained by all (not mentally handicapped) human beings without any culture-driven refinement or schooling. The formation of basic-natural concepts, including basic-natural logical concepts, and attendant basic-natural (monomorphemic) lexicalizations found in natural languages all over the world, takes place at this species-specific level of development.

(b) As part of the human basic-natural ontogenetic cognitive development, an implicit, nondeclarative, and probably universal system of logic comes into being with inbuilt means to restrict any universe of discourse Un to what is needed for any here-and-now purpose at hand. This Un-restriction takes two interrelated forms: (i) conceptual, in that concepts (and the corresponding lexical predicates) are made to function in terms of semantically defined restricted Uns for the negation to select a complement in, and (ii) discoursesemantic, in that, due to the semantics of the main predicate, every successive utterance in a coherent discourse brings about a corresponding restriction of the running Un, again for the negation to select a complement in. A presupposition is a lexically induced semantic condition on a sentence making it suitable for use only in those discourses where that condition is satisfied and unsuitable in others. Presuppositions are thus Un-restrictors par excellence. This basic-natural logical system, complete with inbuilt devices for domain restriction, determines what we take to be "natural intuitions" of entailment, contrariety, and equivalence.

(c) Logic, as a cognitive faculty of individuals, goes through stages not only of ontogenetic but also of cultural development. Culture-induced sophistication leads to higher degrees of explicitness, abstractness, systematicity, and generality, culminating in modern, mathematics-based logic in terms of an abstract, unrestricted $\mathbf{U} \mathbf{n}^{\omega}$.

In this perspective, the study of natural logic becomes an empirical enterprise within the overall theoretical framework of the metalogical relations. When we look at the history of logic in the Western world, we see the progress from minimal to maximal sophistication mentioned in (c) happen before our eyes, and at the same time we see the ecological functionality of "primitive" logic in the context of mundane everyday business mentioned in (b). On these assumptions, the social and cognitive reality of natural languages and the resulting intuitions that serve as empirical data provide an important entrance gate to the hidden mysteries of the logical workings of the mind.

That being so, the question arises of what happens to a logical system when its Un is restricted from $\mathbf{U n}^{\omega}$ to a more restricted universe $\mathbf{U n}^{\mathbf{R}}$. An immediate consequence, which plays a central role in the analysis, is that the negation selects different complements as Un gets more restricted. This is expressed in the fact (already observed by Aristotle) that negative morphological prefixes such as un-, dis-, in-, non-, expressing an opposition between intrinsically positive and negative concepts, typically function within restricted pairs of opposites and thus select complements in highly restricted $\mathbf{U n}^{\mathbf{R}}$ S. For example, my neighbor's dog may not be a Catholic, but he does not count as a non-Catholic: the morphological incorporation of the negation into the composite form non-Catholic automatically restricts the complement of the 
negation to the set of entities that satisfy the presupposition induced by the predicate Catholic which, when used as a count noun, requires presuppositionally that the subject term referent be a human being.

This domain-restrictedness applies likewise to the negation when expressed as an independent word, but with gradations. While negative morphological elements are "minimal" in that they are strictly limited to the minimal $\mathbf{U n}^{\mathbf{R}}$ at hand, the full negation word not is allowed to be used in a "radical" sense, in that it may, in principle, fall back on less-restricted domains as a result of reflection. Thus we can say, $M y$ brother has not come back: he has never been away, where the negation not cancels the presupposition that the speaker's brother has been away. Or indeed we can say My neighbor's dog is not a Catholic, where not breaks through the restricted Un of humans. Yet this license of the negation word not to break through the minimal $\mathbf{U n}^{\mathbf{R}}$ at hand and fall back on an earlier, less restricted, Un is limited by principles of universal grammar. In fact, it seems that the license of being used "radically" is restricted to the negation as a free syntactic element and as a possible addition to any given (assertive) sentence $S$, occupying the so-called "canonical position" in $S$. English has the negation canonically as the head of the auxiliary constituent that goes with the main verb. But when the negation is not the highest sentential operator, or is in any way incorporated into a set expression (idiom), for example, because it is required by a negative polarity item (NPI), or has become part of the morphology, it has no choice but to be "minimal," that is, to select its complement within the minimal $\mathbf{U n}^{\mathbf{R}}$ at hand, being unable to fall back on any earlier, less restricted Un.

5.2 A brief excursion on presuppositions It is not my purpose, in the present study, to elaborate the detailed conditions for the independent negation word not to be used in a marked "radical" sense, restoring the current $\mathbf{U n}^{\mathbf{R}}$ to a less restricted phase in the flow of discourse when a presupposition has been violated (for a full discussion of the presuppositional aspect of this problem, see Seuren [15, pp. 214-313], [18, pp. 334-54]). Yet, in order not to leave the reader completely in the dark as regards this aspect of the analysis, a brief indication is in order of what is at issue. The analysis developed in [15] and [18], and many other of my publications in between these two, implies that the fact that not selects different complements according to whether it is used "minimally" or "radically," requires a truth-conditional distinction between minimal and radical falsity and hence a violation of the Aristotelian principle of strict bivalence. Minimal not by definition preserves presuppositional entailments, since it is these entailments that produce Un-restrictions during discourse (in Seuren [14], my first publication on presupposition theory, I used the term flexible universe of interpretation; see Seuren [16, p. 304]).

Empirical support for the thesis that the distinction between minimal and radical negation is of a logico-semantic and not of a pragmatic nature and is governed by grammatical principles comes from cases like (3) to (5), where not takes the whole rest of the sentence as its scope yet is per se presupposition preserving (!! stands for semantic incongruity with the preceding sentence):

(3) Not only Jack laughed. (!! Jack didn't laugh.)

(4) That her son is gay does not worry the queen. (!! Her son is not gay.)

(5) What worries the queen is not that her son is gay. (!! Nothing worried the queen.) 
In these three sentences, the presuppositions cannot be canceled by not, even though not takes scope over the whole rest of the sentence. In (3), the presupposition that Jack laughed cannot be canceled by not, owing to the fact that not occurs in a noncanonical position. Sentence-initial not thus seems to function as if it were a negative morphological prefix. In (4), the factive presupposition that the queen's son is gay cannot be canceled by not on account of the general but so far unexplained fact that when a factive that-clause stands in its proper subject position and is not extraposed (as in It does not worry the queen that ..., where presupposition canceling is possible), sentence negation cannot cancel the factive presupposition. In (5), the presupposition that something worries the queen is uncancellable by not owing to the fact that the negation over cleft or pseudocleft constructions is necessarily presupposition preserving (for reasons that can only be intuited given the present state of our knowledge). Facts such as these show that minimal and radical not are truth-conditionally distinct. This necessitates the assumption of a trivalent logic, which is best taken to be classically bivalent for any current restricted universe $\mathbf{U n}^{\mathbf{R}}$ but requires the value "radically false" for propositions that violate the boundaries of $\mathbf{U n}^{\mathbf{R}}$. Radical not then generates truth by saying, in effect, that the sentence in question contains a presupposition violation. It is in this sense that we can say (see also note 9 above) that the bivalent logic of the square is maintained for restricted default Uns and that a radical negation operator is called in for nondefault cases where a given restricted $\mathbf{U n}^{\mathbf{R}}$ must be rolled back to a less-restricted $\mathbf{U n}$.

The upshot is that we are looking for a solution to the problem posed by the sentences (1) and (2) in terms of Un-restriction. In particular, we wish to establish a rationale that explains why not all flags are green is restricted in such a way that not only cases where there are no flags but also cases where no flags are green are excluded.

5.3 Polarity division Let us revert now to the question of the logical consequences of Un-restriction as found in natural language. Besides the complement selection of negation, which has just been discussed in some detail, a relation of contrariety in a larger Un may become one of contradictoriness in a more restricted Un: $P \times Q$ becomes $P * Q$ when $\mathbf{U n}$ is restricted to $/ P / \cup / Q /$. Similar changes occur with other logical metarelations. Thus, when $P \vdash Q$ in a larger $\mathbf{U n}$, the entailment is strengthened to the corresponding equivalence $P \equiv Q$ when Un is restricted to $/ P /$ : given that $P \vdash Q$ means that $/ P / \subseteq / Q /$, when $\mathbf{U n}$ is restricted to $/ P /$, $/ P /=/ Q /$; hence $P \equiv Q$ within $\mathbf{U n}^{\mathbf{R}}$. Likewise for the logical independence of $P$ and $Q(/ P /$ and $/ Q /$ mutually partially intersect while $/ P / \cup / Q / \neq \mathbf{U n})$, which turns into subcontrariety when the condition $/ P / \cup / Q / \neq \mathbf{U n}$ is scrapped and Un is restricted to $/ P / \cup / Q /$. In general, equivalence, contradictoriness, subcontrariety, and logical independence depend on the size of Un, whereas entailment and contrariety hold independently of the size of $\mathbf{U n}$, though they may be strengthened to equivalence and contradictoriness, respectively, when $\mathbf{U n}$ is restricted.

Given this, we posit the hypothesis that the cognitive generative process of polarity division is an active force in the coming about of conceptual, including logical, structures.

\section{Polarity Division}

Either pole of a binary opposition of sentence types may, as a result of increased 
cognitive activity, be split up into two mutually exclusive poles. The new, more restricted opposition forms a new, more restricted Un separated from the less restricted Un by a median that fences off the smaller from the larger Un.

Given a binary opposition of sentence types $\langle\mathbf{K}, \neg \mathbf{K}\rangle$ or, as the case may be, $\langle\mathbf{K}, \sim \mathbf{K}\rangle$ (where the symbol $\sim$ stands for negation within some restricted $\mathbf{U n}^{\mathbf{R}}$ ), one, or possibly both of the two poles, usually the nonnegative one $\mathbf{K}$, can give rise to a new binary opposition $\left\langle\mathbf{K}_{\mathbf{1}}, \sim \mathbf{K}_{\mathbf{1}}\right\rangle$, forming a new, more restricted $\mathbf{U n}^{\mathbf{R}}$. Such polarity division is the result of further cognitive activity, such as further analysis or increased knowledge, around the expanded pole K. Cognitively, the original K-pole recedes into the background (often gaining presuppositional status), and the subject now operates in terms of the three contrary poles (vertices) $\mathbf{K}_{1}, \sim \mathbf{K}_{1}$, and $\neg \mathbf{K}$. Yet the original K-pole can be retrieved by conscious, explicit thinking. There is thus a cognitive asymmetry in the new triad of contraries, in that $\mathbf{K}_{1}$ and $\sim \mathbf{K}_{1}$ belong together while $\neg \mathbf{K}$ is the odd one out.

The process is illustrated in Figure 9.a in the ontological terms of valuation spaces and in Figure 9.b in the logical terms of polygonal representations. Once such a

a. Valuation spaces

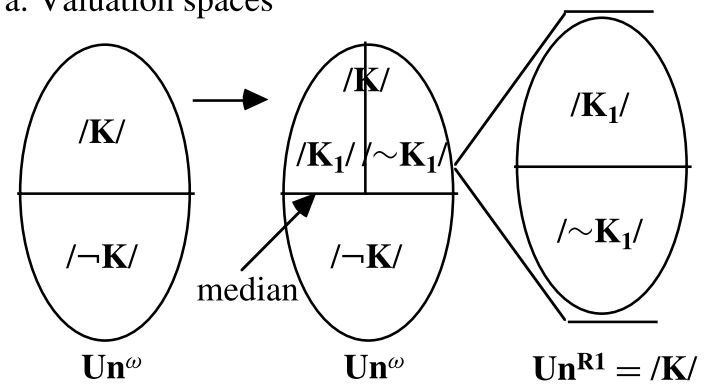

b. Polygonal representations

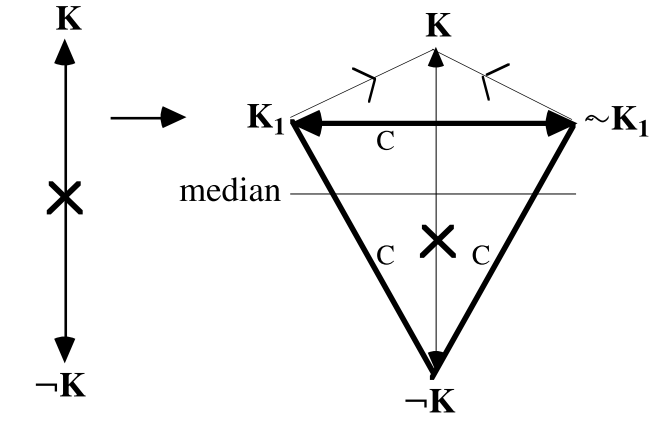

Binary

opposition

Triad of contraries as extension of binary opposition

Figure 9 The transition from a binary to a quaternary opposition, incorporating a triad of contraries. 
a.

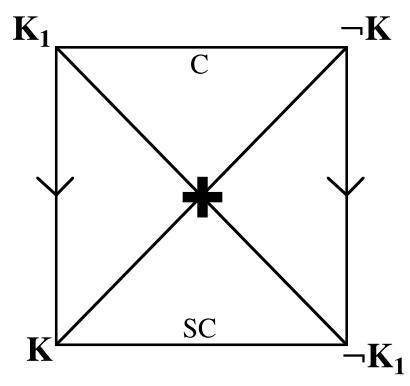

b.

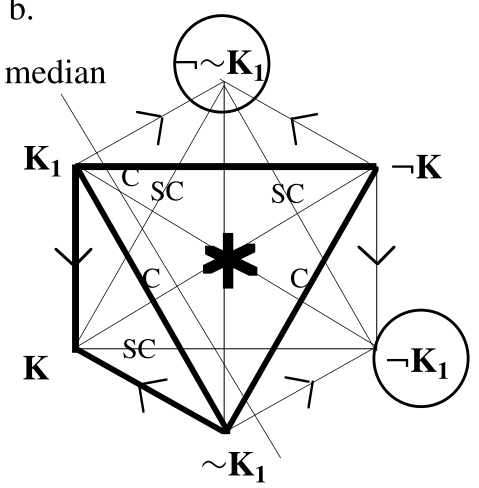

Figure 10 Reduction of Figure 9.b to the traditional square and the Blanché hexagon.

division has taken place, the boundary between the two original opposites fencing in the new $\mathbf{U n}^{\mathbf{R}}$ is called the median.

Assuming that the point of departure is $\mathbf{U n}^{\omega}$, so that the complement of $/ \mathbf{K} /$ is $/ \neg \mathbf{K} /$, and assuming that $\mathbf{K}$ is split up into $\mathbf{K}_{1}$ and $\sim \mathbf{K}_{1}$, it follows that both $\mathbf{K}_{1}$ and $\sim \mathbf{K}_{1}$ entail $\mathbf{K}$ and that $\mathbf{K}_{1}$ and $\sim \mathbf{K}_{1}$, which are contraries in $\mathbf{U} \mathbf{n}^{\omega}$, are contradictories in the more restricted $\mathbf{U n}^{\mathbf{R} 1}$ formed by $/ \mathbf{K} /$. Figure 9.b shows that the original binary opposition $\langle\mathbf{K}, \neg \mathbf{K}\rangle$ has been extended to a quadrilateral structure, henceforth called a kite, which, in the original terms of $\mathbf{U} \mathbf{n}^{\omega}$, incorporates a triangle of contraries. At this stage of development, the triad $\left\langle\mathbf{K}_{\mathbf{1}}, \sim \mathbf{K}_{\mathbf{1}}, \neg \mathbf{K}\right\rangle$ is cognitively prominent, and the original $\mathbf{K}$-vertex has receded into the background, from where it is retrievable by intellectual reflection.

One notes that the kite only contains the relations of entailment, contrariety, and contradiction, not the highly counterintuitive relation of subcontrariety. When the kite of Figure 9.b is rearranged into the square format, as in Figure 10.a, one finds that there is no place for a $\sim \mathbf{K}_{1}$-vertex, as the square format has only one contrariety relation, that between $\mathbf{K}_{1}$ and $\neg \mathbf{K}$. By contrast, the hexagon format of Figure 10.b allows for all relations of Figure 9.b to be represented but adds a few more, making the picture complete, with the $\sim \mathbf{K}_{1}$-vertex standing for the Blanché $\mathbf{Y}$-vertex and the $\neg \sim \mathbf{K}_{\mathbf{1}}$-vertex for the Blanché $\mathbf{U}$-vertex. The kite of Figure 9.b is present in the quadruple $\left\langle\mathbf{K}_{\mathbf{1}}, \mathbf{K}, \sim \mathbf{K}_{\mathbf{1}}, \neg \mathbf{K}\right\rangle$ of Figure 10.b. The two vertices that have been added to the kite are $\neg \mathbf{K}_{\mathbf{1}}$ and $\neg \sim \mathbf{K}_{\mathbf{1}}$, which have been circled in Figure 10.b. The reason for singling out the vertices $\neg \mathbf{K}_{\mathbf{1}}$ and $\neg \sim \mathbf{K}_{\mathbf{1}}$ (Blanché's $\mathbf{U}$-vertex) is that these two select a complement outside their $\mathbf{U} \mathbf{n}^{\mathbf{R}}$. This is not so for the $\neg \mathbf{K}$-vertex, which selects its complement within the higher order $\mathbf{U n}$, perhaps $\mathbf{U} \mathbf{n}^{\omega}$, but $\neg \mathbf{K}_{\mathbf{1}}$ and $\neg \sim \mathbf{K}_{\mathbf{1}}$ per se reach back into a $\mathbf{U n}$ of a higher order, as is signaled by the combination of $\neg$ and a subscript under $\mathbf{K}$.

This is relevant for the following reason. It has been observed that there is a virtually universal lexical asymmetry in natural languages in that there are basic (monomorphemic) lexicalizations for the $\mathbf{A}-$-, I-, and $\mathbf{E}(=\sim \mathbf{I})$-vertices (resp., all, some, and no in English) but not for the $\mathbf{O}$-vertex (not all). The first author known to have made this observation was Thomas Aquinas (1224/5-1274) in his Expositio 
Peryermeneias (liber 1, lectio 10, nota 13, www.corpusthomisticum.org/cpe.html; see [6, Chapter 4], [5, p. 253], Jaspers [9, p. 15]), followed some time later by Blanché [1, pp. 95-6], Horn [6, Chapter 4], [5, pp. 252-62], Löbner [12, p. 95], Levinson [11, pp. 69-71], Jaspers [9], Seuren [18, pp. 114-21], and others. The $\mathbf{O}$-vertex is represented in Figure 10. b by $\neg \mathbf{K}_{\mathbf{1}}$, which, as we have seen, contravenes the stipulated restriction that, in basic cognition, the negation selects its complement within the nearest $\mathbf{U n}$. That the $\mathbf{U}\left(=\neg \sim \mathbf{K}_{\mathbf{1}}\right)$-vertex commits the same sin was observed recently by Dany Jaspers, in the context of renewed interest in the traditional square and in the works of Robert Blanché [1], [2] and a few other forgotten authors, such as Paul Jacoby [7], [8] and Augustin Sesmat [13], who were unhappy with the summary dismissal of traditional predicate logic by Bertrand Russel and modern mathematical logicians in general.

The pragmatic answer to this lexical asymmetry (see [6, Chapter 4], [11, pp. 69-71]) amounts to the following chain of reasoning. Exclusive some (the Y-vertex) results from the Gricean first maxim of quantity (see [3, p. 45]) reinforced by scalar implicatures (see above). Since exclusive some is pragmatically equivalent with some-not (the $\mathbf{O}$-vertex), and since lexicalization generally favors positive rather than negative concepts, there is no need for a lexicalization of the $\mathbf{O}$-vertex. However, it has been argued above that this pragmaticist solution is insufficient. We may add here that this answer also fails to explain the universal nonlexicalization of the $\mathbf{U}$-vertex. Finally, since the nonlexicalization of the $\mathbf{O}$ - and $\mathbf{U}$-vertices is just one instance of a general feature of the lexicons of languages as a whole, which systematically never lexicalize negatives across medians, and since the pragmatic answer given by Horn and Levinson has no bearing on the nonlogical cases of systematic nonlexicalization, it seems that this pragmatic answer must be rejected. ${ }^{19}$

The answer given in Seuren [18, pp. 114-21] is likewise insufficient. Seuren argues that in the basic-natural triad of contraries no is not a lexicalization of "not-(exclusive)-some," which would yield the meaning "all or no" (i.e. the U-vertex). Therefore, there is no reason to expect any lexicalization for "not-all" in the O-vertex: the problem does not arise. While this is in itself correct, it fails to explain the corresponding lexical gaps systematically found outside the various logical systems.

The answer proposed here is based on Jaspers [9]. Jaspers found the opening to the solution elaborated here, in that he proposed a cognitive hierarchy in the vertices of the classic square such that $\mathbf{I}$ is cognitively opposed to not-I in a larger Un but A is cognitively opposed to not-A in a smaller Un defined by $\mathbf{I}$, excluding not-I. He then proposed a lexicalization principle according to which lexicalizations of complements cannot cross a border into a larger Un but have to stay within their own (sub)division. This prevents a lexicalization of $\neg \mathbf{A}$ and thus of $\mathbf{I}^{*}$. This inspired hypothesis seems to be the correct answer. It covers the nonlexicalization of both the $\mathbf{O}$ - and the $\mathbf{U}$-vertices and of corresponding vertices for all lexically induced opposition relations and thus has by far the greatest explanatory power of all explanations proposed so far. Moreover, it allows for the design of a model for the ontogenesis of natural logic.

Consider the cognitive state of a human being around birth. The very first sensory impression will result in the sensation that "there is something out there," as against "there is nothing." Repeated events of this nature will lead to the discovery of qualitative differences or properties, such as between moving and still entities, between 


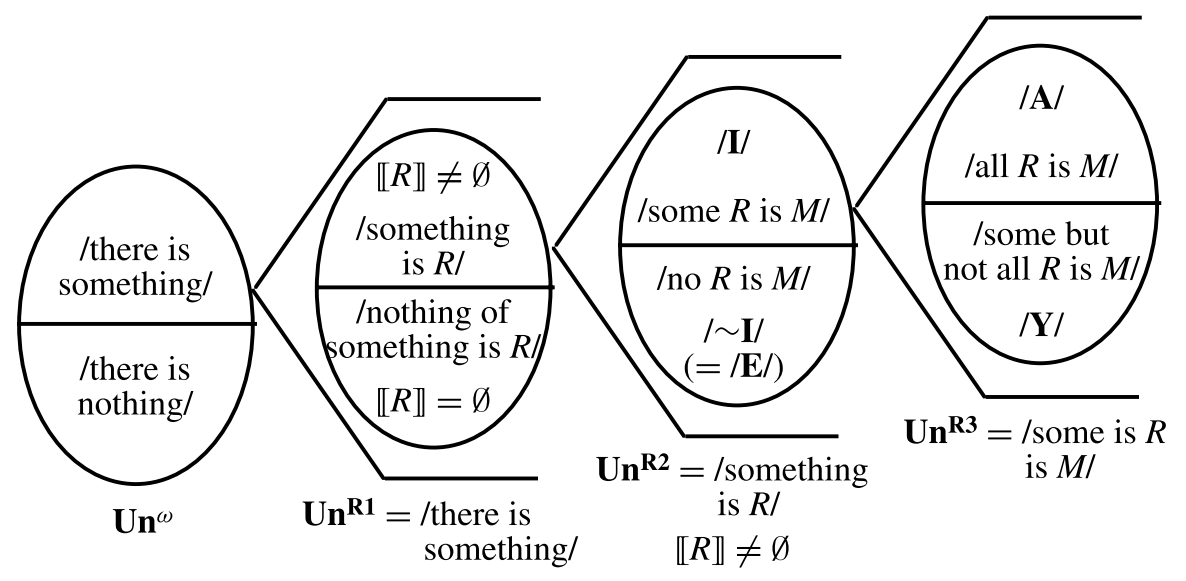

Figure 11 The ontogenesis of predicate logic in terms of successive Un-restriction.

individual entities and backgrounds, between entities that feed and those that do not, between noise and silence, and so on. At a further stage, the infant will start generalizing over properties (that is, she or he will start quantifying), noticing that some entities with property $R$ also have property $M$, or that no entity $R$ also has property $M$. Then, the infant will discover that, so far, all, or not all, entities $R$ also have property $M$. This process is schematically predicted in Figure 11.

For example, the infant finds that some entities "out there" produce noises but others do not. This is stage b in Figure 12, repeated over and over for a number of entities and a number of properties. At stage $\mathrm{c}$, the infant gets sensitized to the fact that one particular entity not only makes noises but also feeds and causes a sensation of well-being, while other entities do other things. That is, in virtue of an inbuilt program, the infant starts to spread properties over individuals, inductively categorizing the spread patterns into the binary concepts of (inclusive) "some" versus its opposite "none" in propositional thought structures, thus creating the types I and $\sim \mathbf{I}$ (see Figure 12.c). At stage d, the I-pole of the $\mathbf{I}$ vs $\sim \mathbf{I}$ opposition is expanded into a further opposition between a tentatively inductive "all" (A) and an empirically established "not-all" ( $\sim \mathbf{A} \equiv \mathbf{Y})$, whereby both $\mathbf{A}$ and $\sim \mathbf{A}$ entail $\mathbf{I}$, causing ("undue") existential import. Figure 12.e represents a potential further subdivision of the $\mathbf{A}-\mathbf{Y}$ opposition creating a hypothetical $\mathbf{U} \mathbf{n}^{\mathbf{R} 4}$ in which not-all (Y) has been split up into many $R$ is $M$ and few (= not many) $R$ is $M$. Whether this is indeed a valid extension of the system cannot be discussed here, but, given our present insights, it cannot be excluded.

This development is schematically represented in Figure 11, which shows the successive restrictions from $\mathbf{U} \mathbf{n}^{\omega}$ to $\mathbf{U} \mathbf{n}^{\mathbf{R} 3}$, at which point the concepts expressed in I-, $\sim \mathbf{I}-, \mathbf{A}-$, and $\mathbf{Y}(=\sim \mathbf{A})$-type sentences have come into being. The existential import of these sentence types and the subaltern entailment $\mathbf{A} \vdash \mathbf{I}$ are now seen to result from the cognitive process we have called polarity division. Figure 12 shows the same in terms of polygonal representations (kites).

One notes that what is traditionally called the $\mathbf{E}$-vertex represents $\sim \mathbf{I}$, which selects its complement within $\mathbf{U n}^{\mathbf{R} 2}$, not $\neg \mathbf{I}$, which does so within $\mathbf{U n}^{\omega}$. While $\sim \mathbf{I}$ en- 


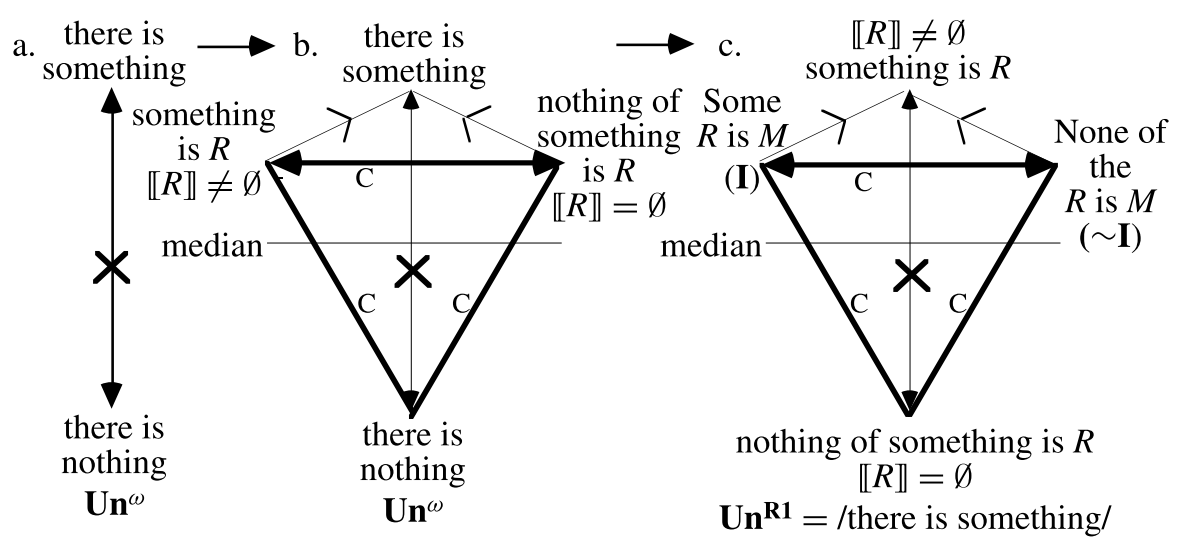

(I)

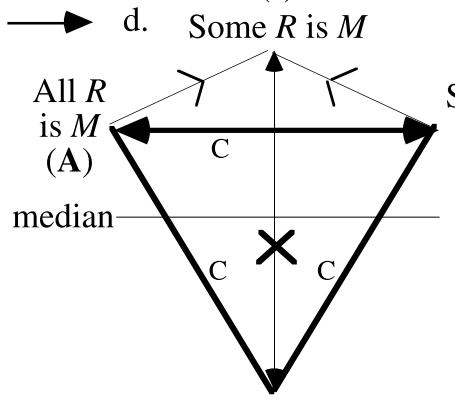

None of the $R$ is $M$

$(\sim$ I)

$\mathbf{U n}^{\mathbf{R 2}}=/$ something is $R /$ $\llbracket R \rrbracket \neq \varnothing$
(Y)

?? $\longrightarrow$ e. $\quad$ Some but not all $R$ is $M$

Some but

not all

$R$ is $M$

$(\mathbf{Y})$

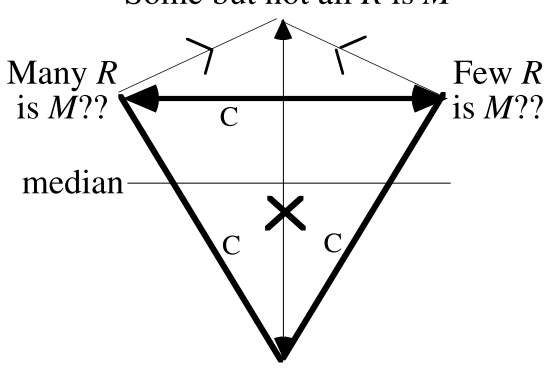

All $R$ is $M$

(A)

$\mathbf{U n}^{\mathbf{R 3}}=/$ some is $R$ is $M /$

Figure 12 The ontogenesis of predicate logic in terms of logical diagrams.

tails that $\llbracket R \rrbracket \neq \emptyset, \neg \mathbf{I}$ has no such entailment. We may take it that natural language default not represents $\sim$, not $\neg$. Through the ages, logicians have not observed this distinction and have, unwittingly, created a new, artificial not doing its work within the philosophically constructed $\mathbf{U} \mathbf{n}^{\omega}$. Yet in the square, the vertex denoted by $\mathbf{E}$ or not-I entails that $\llbracket R \rrbracket \neq \emptyset$, but in systems where space 4 is taken into account no such entailment exists.

The procedure of polarity division has thus generated the quadrilateral system represented in Figure 13.a, which has the kite structure of Figure 9.b. Further logical reflection in terms of $\mathbf{U} \mathbf{n}^{\omega}$ adds the $\neg \mathbf{A}$ and $\neg \mathbf{Y}(=\mathbf{U})$ vertices shown in Figure13.b, both carrying the negation $\neg$. It is precisely these two added vertices that escape basic-natural lexicalization. The solution proposed in the first instance in Jaspers [9] is thus dramatically confirmed. ${ }^{20}$

It should be noted that, in this new, most basic-natural system of predicate logic, the minimal negation $\sim$ selects its complement within the restricted $\mathbf{U n}^{\mathbf{R}}$ of its argument. That is, / $\sim \mathbf{I} /$ is the complement of $/ \mathbf{I} /$ within the opposition I vs $\sim \mathbf{I}$, while 
a.

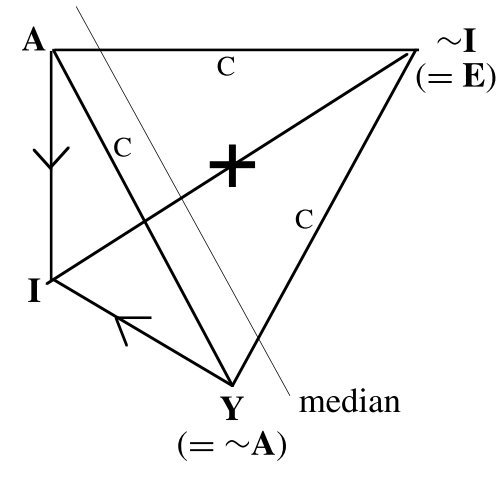

b.

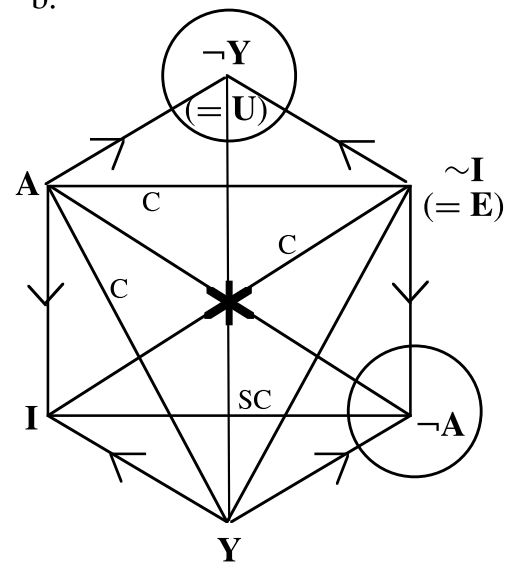

$\mathbf{U n}^{\mathbf{R} 2}=$ something is $R: \llbracket R \rrbracket=\emptyset$

Figure 13 The basic-natural kite structure, extended to the hexagon by reflection.

a.

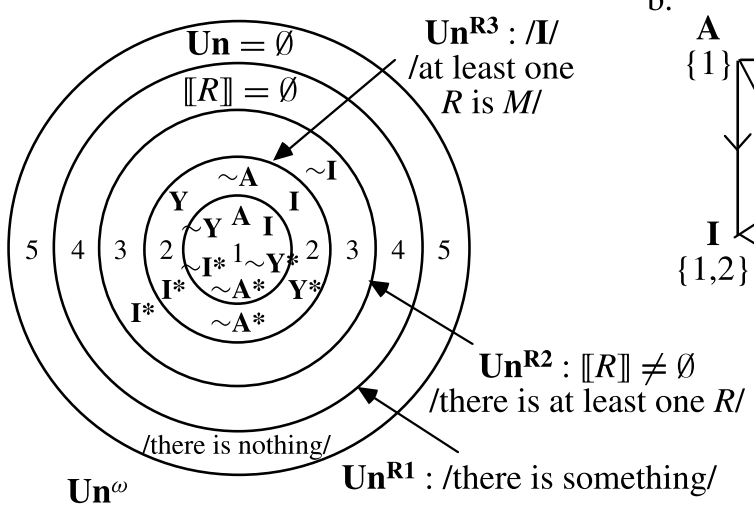

b.

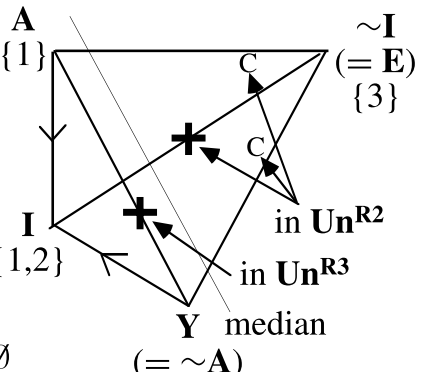

$\{2\}$

/there is nothing or there is something/

Figure 14 VS-analysis and corresponding kite of the most basic natural predicate logic.

/ $\sim \mathbf{A} /$ is the complement of $/ \mathbf{A} /$ within the opposition $\mathbf{A}$ vs $\sim \mathbf{A}(=\mathbf{Y})$, jointly forming $/ \mathbf{I} /{ }^{21}$ This produces the VS-analysis of Figure 14.a, which has an added space 5 for the logically possible situation, not considered earlier, of a null universe. Here spaces 5 and 4 are not filled in because only the restricted negation $\sim$ is considered, space 3 is filled in only for (the minimal negation of) $\mathbf{I}$ and for $\mathbf{I}^{*}$, and the sentence types $\mathbf{A}, \mathbf{Y}, \mathbf{A}^{*}, \mathbf{Y}^{*}$, and their minimal negations are valued only for spaces 1 and 2 .

In Figure $14, \mathbf{A}, \mathbf{Y}$, and $\sim \mathbf{I}$ are still contraries, since $/ \mathbf{A} /=\{1\}, / \mathbf{Y} /=\{2\}$, and $/ \sim \mathbf{I} /=\{3\}$, though the contrariety of $\mathbf{A}$ and $\mathbf{Y}$ has been strengthened to contradiction due to polarity division, and the subaltern entailment still holds for $\mathbf{A} \vdash \mathbf{I}$, but 
that, therefore, the entailment $\mathbf{A}^{*} \vdash \mathbf{I}^{*}$ should likewise hold cannot be expressed, since the $\mathbf{U n}^{\mathbf{R}}$ for universally quantified sentences consists of just the spaces 1 and 2 , while $\mathbf{A}^{*}$ is true only in space 3 , leaving perfectly grammatical sentences of the type all Romans are not mortal without a semantics, the only remedy being a negativization of the predicate, all Romans are nonmortal, which is an A-type sentence. This is why it took some nontrivial reflection to arrive at the entailment $\mathbf{A}^{*} \vdash \mathbf{I}^{*}$, expressible in terms of the Blanché hexagon shown in Figure 3 above. For the same reason, the conversions are expressible only partially. Figure 14 shows that the equivalences $\mathbf{A} \equiv \sim \mathbf{I}^{*}$ and $\mathbf{I} \equiv \sim \mathbf{A}^{*}$ are expressed, since $/ \mathbf{A} /=/ \sim \mathbf{I}^{*} /=\{1\}$ and $/ \mathbf{I} /=/ \sim \mathbf{A}^{*} /=\{1,2\}$. But the equivalences $\mathbf{A}^{*} \equiv \sim \mathbf{I}$ and $\sim \mathbf{A} \equiv \mathbf{I}^{*}$ cannot be expressed. These gaps had to be filled in by further logical reflection, ultimately leading to the Blanché hexagon. Without such reflection, $\sim \mathbf{A} \equiv \mathbf{Y}$, since $/ \sim \mathbf{A} /=/ \mathbf{Y} /=\{2\}$.

The logic displayed in Figure 14 is, so to speak, a system with sliding panels, which surely makes this system logically unsound, as it is not valid for all possible situations, but no less interesting for that. I take it that this system is the most basic system of natural logic found in human nature-more basic even than the Hamiltonian triad. It is no exaggeration to say that the history of logic can be seen as a progressive filling in, interrupted by setbacks, for all the sentence types involved, of the gaps left by the VS-analysis of Figure 14.a. This labor involved the search for a unified, nonflexible $\mathbf{U} \mathbf{n}^{\omega}$ for predicate logic as a whole, covering all possible situations and thus attaining the status of analytical necessity. The classic square of opposition unified its Un and filled in all the gaps up to space 4 (where $\llbracket R \rrbracket=\emptyset$ ) for the sentence types $\mathbf{A}$ and $\mathbf{I}$ plus their internal and external negations. The Hamiltonian triad did the same for the sentence types $\mathbf{A}, \mathbf{Y}$, and $\mathbf{E}(=\sim \mathbf{I})$ plus their internal and external negations. Both standard modern logic and Aristotelian-Abelardian logic unified their Un and filled in all the gaps including space 5 (provided the logic is formulated in terms of generalized quantifiers), ${ }^{22}$ thus arriving at the one and only $\mathbf{U n}^{\omega}$ for the sentence types $\mathbf{A}$ and $\mathbf{I}$ plus their internal and external negations. The Blanché hexagon, when extended to cover cases where $\llbracket R \rrbracket=\emptyset$ and formulated in terms of generalized quantifiers, finishes the task for the sentence types $\mathbf{A}$, $\mathbf{I}$, and $\mathbf{Y}$ plus their internal and external negations.

This not only solves the problem of the intuitively felt equivalence of the sentences (1) (not all flags are green) and (2) (some flags are green and some flags are not green) quoted above but also the general problem of the intuitive clashes of natural logical intuitions with standard modern predicate logic. All it took, in hindsight, was the construction of the logic spontaneously developing in human beings, which has been shown to be incomplete but extendable to at least two universally valid forms of predicate logic: standard modern and Aristotelian-Abelardian predicate logic.

\section{Some Final Observations}

A recurrent feature of quadrilateral lexical systems such as those shown in Figure 13.a is that the $\mathbf{U n}^{\mathbf{R}}$ that acts as an umbrella for a new opposition recedes to presuppositional status. Thus, the I- and $\sim$ I-vertices of Figure 12.d presuppose that $\llbracket R \rrbracket \neq \emptyset$, and so do the subsequent subdivisions $\mathbf{A}$ and $\sim \mathbf{A}$. How general this phenomenon is in lexicons as a whole is a question that has not yet been investigated 
systematically. But it can be observed at this point that similar recessions to presuppositional status are found in, for example, the items of the complex crime, illegal, legal, offense (see note 19), which all presuppose that there is penal law. Likewise for the complex consisting of bachelor, single, spinster, married, which all presuppose a common $\mathbf{U n}^{\mathbf{R}}$ where the subject referents of these predicates are adult humans and where there is a socially sanctioned institution of marriage.

A further recurrent, but by no means universal, feature is that lexical items in the I-position tend to be of a more technical or academic nature and thus outside or above the register of common usage. Thus, the word sibling, in the I-position of the kite, belongs to a more legal or technical register than the words brother and sister, which occupy two vertices of the triad of contraries 〈brother, sister, parent $\rangle$. Likewise for animal, which, in the I-position, belongs to an academic register covering human and nonhuman animals, while in the $\mathbf{Y}$-position it covers only nonhuman animals. This can be ascribed to the fact, mentioned earlier, that the pole which is split up into two subpoles, thereby creating a more restricted Un, recedes into the background so that the subject now operates in terms of the remaining triad of contraries. The "forgotten" pole is then still retrievable, but the retrieval requires conscious reflection. As regards the quantifiers, the hypothesis is thus that lexicalization takes place at the level described in Figure 12.d for the vertices of the triad of contraries $\langle\mathbf{A}, \mathbf{Y}, \sim \mathbf{I}\rangle$. The I-vertex was retrieved by Aristotle, through conscious reflection.

Often words occupying the $\mathbf{Y}$ - and I-positions of the kite structures involved are phonologically identical, thus creating a systematic ambiguity. The prototypical example is, of course, the culturally created ambiguity of the quantifying word some and its equivalents in all languages spoken in sufficiently sophisticated societies. Outside logic, the same is found with, for example, the word animal, which has its wider, academic sense in the I-position and its common, more restricted sense in the Y-position of the kite. Likewise for the word sea, which is opposed to ocean when used in the $\mathbf{Y}$-position but covers both (minimal) sea and ocean in the I-position. (To say that a ship is at sea does not imply that it is not in an ocean.) Or the word gay, which comprises both male and female gays in its use in the I-position but only male gays in the $\mathbf{Y}$-position. The English word ground occupies the I-position (where it includes the sea surface: the grounding of planes includes seaplanes), but the closely related land occupies the Y-position (see Seuren and Jaspers [21]). The Romance languages, however, have the same word (terra or terre) for both meanings. Why this should be so is not clear beyond a speculative level of thought.

\section{Conclusion}

It thus seems that an uncluttered cognitive perspective on logic has a considerable explanatory force, especially, but not exclusively, with regard to the clash between standard modern predicate logic on the one hand and natural logical intuitions on the other. The analyses presented here undercut the widely accepted but fallacious view that Gricean pragmatics suffices as an account of this clash. They also show that cognition, though subject to metaphysical necessity and to the laws of physics, has managed to find its own functionally advantageous path through the maze of possibilities afforded by these two great overarching sets of constraints. The present study is an attempt at unveiling the hitherto mysterious selections made and the systems 
developed by human cognition in its effort to cope effectively with the big world "out there."

Cognition as it exists and functions in individuals and, by extension, in societies and coherent groups of humans in general can only be an object of scientifically explanatory inquiry if it is recognized that it has structures and principles of its own, many of which are formally definable. Cognition does not simply emulate mathematics, nor is it a processing machine that receives material input and produces material output according to the laws of physics. This quasi-objectivity fallacy has stymied progress in the human sciences for too long. The recognition of the specifically human factor in cognitive processes is a prerequisite for the human sciences to become truly scientific.

\section{Notes}

1. A conspicuous case, not discussed in the present study, where the Gricean maxims are seen to be irrelevant is the following. Sentence (ia) is immediately felt to be equivalent with sentence (ib). Yet (iia) is not at all immediately felt to be equivalent with (iib), even though both (i) and (ii) are instances of the De Morgan's conversion laws:

(i) a She doesn't like planes or trains.

b She doesn't like planes and she doesn't like trains.

(ii) a She doesn't like planes and trains.

b She doesn't like planes or she doesn't like trains.

Here, the Gricean maxims have nothing to say. The cases (i) and (ii) are not further analyzed here, as the present study is restricted to predicate logic. For a discussion, see Seuren [18, pp. 108-14].

2. Bivalence is not a necessity. It is perfectly possible to have, say, three truth values, for example, true with two kinds of falsity, false I and false II, as in my presuppositional logic. Or one can have an infinity of truth values with gradual transitions, as in Zadeh's so-called "fuzzy" logic (see Zadeh [23]). And other variations on this theme are viable.

3. It is possible to define the metalogical relations in terms of the truth-functional connectives. Thus, $P \vdash Q$ is equivalent to $\Vdash(P \rightarrow Q)$, where $\Vdash$ stands for "for all possible situations." $P \times Q$ is thus definable as $\Vdash \neg(P \wedge Q), P \simeq Q$ as $\Vdash(P \vee Q), P * Q$ as $\Vdash(P \vee Q)$ ( $\vee$ standing for the exclusive $\vee$ ), and $P \equiv Q$ as $\Vdash(P \rightarrow Q) \wedge(Q \rightarrow P)$. This notation in terms of the truth-functional connectives is not used here, as it is assumed that the use of single symbols for each metalogical relation makes for easier reading.

4. In the figures, arrows stand for entailment, $\mathrm{C}$ for contrariety, crosses, stars or $\mathrm{CD}$ for contradictoriness, $=$ for logical equivalence, $\mathrm{SC}$ for subcontrariety, and the absence of a connecting line for logical independence.

5. It will become clear below that a distinction must be made between the classical negation $\neg$, which selects a complement in the unrestricted universe of discourse $\mathbf{U n}^{\omega}$, and the minimal negation $\sim$, which does so within some restricted universe of discourse $\mathbf{U n}^{\mathbf{R}}$.

6. The traditional notation is $\mathbf{A}$ for all $R$ is $M$, $\mathbf{I}$ for some $R$ is $M, \mathbf{E}$ for $n o R$ is $M$, and $\mathbf{O}$ for not all $R$ is $M$. This notation is due to the Aristotle commentator Boethius, whose rendering of Aristotelian predicate logic determined the history of this subject throughout the 
Middle Ages and beyond, until \pm 1900 . Boethius took the vowels $\mathbf{A}$ and $\mathbf{I}$ from the Latin word AffIrmo (I affirm) and the vowels $\mathbf{E}$ and $\mathbf{O}$ from Latin $n E g O$ (I deny). It served well throughout the Middle Ages and later, but our analysis requires a more refined notation, which makes the position of the negation explicit: $\neg$ for the external negation and $*$ for the internal negation.

7. I adopt the standard notation of double bracketing to denote the extension of a predicate: for any predicate $Z, \llbracket Z \rrbracket$ is the set of elements for which $Z$ delivers truth.

8. It is still widely believed that Aristotle created the square with the conversions and thus with the fault of UEI. This belief is false (see [18, pp. 149-55] for a detailed account). It has been well known for some time among serious historians of logic that Aristotle did not commit the error of UEI, but this insight has not been widely publicized. That Abelard restored Aristotle's predicate logic to its pristine, error-free form is my own contribution.

9. Another way of making ABPL sound, envisaged neither by modern logicians nor by Aristotle or Abelard, consists in keeping the square as it is, complete with conversions and UEI, and treating the condition that $\llbracket R \rrbracket \neq \varnothing$ as a presupposition. (More is said below in Section 5.2.) Propositions not satisfying that presupposition are relegated to a nondefault region of logic which makes them radically false, a more drastic form of falsity than ordinary or minimal falsity requiring a marked, discourse-correcting radical negation to yield truth. The default part of the system then stays classically bivalent, but the nondefault part adds radical falsity as a third truth value. It is claimed in [18] and earlier publications that this is how UEI is solved in natural language.

10. Another element in Hamiltonian logic is the so-called quantification of the predicate, which allows for sentences such as all Romans are some mortals or some Romans are all mortals. Since this device of double quantification does not seem to yield any gains, it is not discussed here.

11. The term and the notion of valuation space stem from Van Fraassen [22], where, however, the potential of that notion remained underdeveloped.

12. The number of possible situations explodes exponentially as more sentences are added. With 10 logically independent sentences, we have $2^{10}$ or 1024 possible situations. With 25 sentences there are $2^{25}$ or $33,554,432$ possible situations. How to define the astronomical number of possible situations with an infinite set of sentences is a question I am happy to leave to arithmeticians. It has no relevance in the present context.

13. Most logicians using generalized quantification take the $R$-predicate as the first or subject and the $M$-predicate as the second or object term. Yet the grammatical mapping process from the logical language here described to the surface form of sentences is crucially simplified if the $M$-predicate is taken to be the subject and the $R$-predicate the object term (see [18, p. 55]). Moreover, the logical notion of scope is now definable for all cases as the subject ( $=$ first) term of the predicate.

14. No distinction is made here between first-order and higher-order sets: the variables $X$ and $Y$ stand for higher-order sets of any rank. In most practical cases, however, $X$ and $Y$ will be first-order sets. 
15. Chapter 8 in [20] specifies an algorithmic procedure leading from all possible settheoretic relations between $\llbracket R \rrbracket$ and $\llbracket M \rrbracket$ to the distribution over the spaces 1 to 4 .

16. I am indebted to Hans Smessaert, of Katholieke Universiteit Leuven (pc), for the arrangement of the vertices in Figure 4.b and later polygonal representations. In previous publications I have had different arrangements, but the one given here is clearly the correct one, as it shows better than other arrangements the symmetries in the system. For the logic as such, the arrangement makes no difference. Note that in the minimalist representation of Figure 4.c the symbol CD has become unnecessary, as all relations of contradictoriness pass through the center. This is, in effect, a test for the minimalness of polygonal representations, as is confirmed in later figures.

17. Jespersen [10, p. 85] picks the example of not lukewarm, which "in most cases means less than lukewarm, i.e. cold or something between cold and lukewarm." This observation is open to doubt, as lukewarm seems to have a directionality from warm to cold: The coffee was still lukewarm. This directionality seems due to a presupposition induced by lukewarm to the effect that what is said to be lukewarm should be warmer: a lukewarm reception. Not lukewarm would thus preferably suggest "warm" rather than "cold."

18. This fact is recognized in Seuren [18, pp. 99-101], which was written when the analysis proposed in the remainder of the present paper had not yet been developed. The tentative solution proposed in [18] for cases like (1) and (2) rests on the assumption of an underlying default topic-comment structure in not-all sentences, which would induce a presupposition of existence and thus rule out the no-cases (the $\neg \mathbf{I}$-vertex). In the light of the new analysis presented here, I now consider this answer to be superseded.

19. For the universal nonlexicalization of $\mathbf{O}$ - and $\mathbf{U}$-vertices across languages, see Seuren and Jaspers [21]. To give one example out of many, crime (the A-vertex) entails illegal (the I-vertex); illegal is the Un-restricted contradictory (contrary without Un-restriction) of legal (the $\sim \mathbf{I}$-vertex); no lexicalization exists for "not-crime" (the $\neg \mathbf{A}$-vertex), covering both minor wrongdoings and legal acts, but there is the lexicalization offense (the Y-vertex) for what constitutes a breach of the law but is not a crime, such as a traffic offense. Nor is there a lexicalization for the $\mathbf{U}$-vertex, meaning "either a crime or legal."

20. An analogous analysis should be given of the cognitive ontogenesis of propositional logic, which will then probably be seen to make explicit the well-known relation between some and or on the one hand, and between all and and on the other. That work, however, has not been carried out so far. Despite this incompleteness, it seemed opportune to offer the results for predicate logic for publication, pending further developments.

21. The notation used for the minimal negation $\sim$ is deficient in that $\sim$ should be formally indexed for the restricted domain it selects its argument's complement in. I have not introduced such indexing since to do so would require a fairly complex mechanism, which would add to the length of the present study and might thus detract from the main issues. A formally more precise notation will no doubt follow. I trust that, despite this formal deficiency, the gist of the analysis will be clear.

22. As is well known, when expressed in terms of the material implication for all and the conjunction for some, as proposed by Russell and common practice during the whole 
of the twentieth century, standard modern predicate logic does not work for space 5, reserved for a null universe, since variable substitution is impossible in space 5. This problem is solved by the language of generalized quantification, whose semantics is based on set theory and thus does not require any mechanism of variable substitution. In a null Un, the classic example of a logically necessary truth, all bachelors are unmarried, loses that status in all logics where the universal quantifier requires nonnullness of the $R$-class. When cast in terms of the material implication, with its standard truth table, that is, as "for all $x$, if $x$ is a bachelor, then $x$ is unmarried," the sentence is uninterpretable due to lack of substitution values. When cast in the terms of generalized quantification, as "the set of all bachelors is included in the set of all unmarrieds," the sentence regains its status of analytical necessity.

\section{References}

[1] Blanché, R., "Sur l'opposition des concepts," Theoria, vol. 19 (1953), pp. 89-130. 500, 503, 515, 522

[2] Blanché, R., Structures intellectuelles: Essai sur l'organisation systématique des concepts, edited by J. Vrin, Librarie Philosophique, Paris, 1966. 500, 503, 515, 522

[3] Grice, H. P., "Logic and conversation," pp. 41-58 in Speech Acts, edited by P. Cole and J. L. Morgan, Academic Press, New York, 1975. 515, 522

[4] Hamilton, W., "Lectures on metaphysics and logic, IV" in Lectures on Logic. Vol. II, edited by H. L. Mansel and J. Veitch, reprint of the 1886 edition, Michigan Historical Reprint Series, Scholarly Publishing Office, University of Michigan Library, Ann Arbor, 2005. 506

[5] Horn, L. R., A Natural History of Negation, University of Chicago Press, Chicago, 1989. MR 0978195. 516, 522

[6] Horn, L. R., "On the semantic properties of logical operators in English," Ph.D. dissertation, University of California, Los Angeles, Los Angeles, Calif., 1972. 516, 522

[7] Jacoby, P., "A triangle of opposites for types of propositions in Aristotelian logic," The New Scholasticism, vol. 24 (1950), pp. 32-56. 522

[8] Jacoby, P., "Contrariety and the triangle of opposites in valid inferences," The New Scholasticism, vol. 34 (1960), pp. 141-69. 522

[9] Jaspers, D., "Operators in the lexicon: On the negative logic of natural language," Ph.D. thesis, Leiden University, LOT, Utrecht, Netherlands, 2005. 522, 524

[10] Jespersen, O., Negation in English and Other Languages, vol. 1.5 of Det Kgl. Danske Videnskabernes Selskab, Historisk-filologiske Meddelelser, Høst, Copenhagen, 1917. 515, 530

[11] Levinson, S. C., Presumptive Meanings: The Theory of Generalized Conversational Implicature, MIT Press, Cambridge, Mass., 2000. 515, 522

[12] Löbner, S., Wahr neben Falsch: Duale Operatoren als die Quantoren natürlicher Sprache, Niemeyer, Tübingen, 1990. 504, 522

[13] Sesmat, A., Logique, II: Les raisonnements, la logistique, Hermann, Paris, 1951. 522

[14] Seuren, P. A. M., "Taaluniversalia in de transformationele grammatika," Leuvense Bijdragen, vol. 61 (1972), pp. 311-70. 518

[15] Seuren, P. A. M., Discourse Semantics, Blackwell, Oxford, 1985. 518

[16] Seuren, P. A. M., A View of Language, Oxford Univ. Press, Oxford, 2001. 518

[17] Seuren, P. A. M., "The importance of being modular," Journal of Linguistics, vol. 40 (2004), pp. 593-635. 500

[18] Seuren, P. A. M., The Logic of Language: Language from Within, Vol. II, Oxford Univ. Press, Oxford, 2010. 504, 505, 513, 514, 518, 522, 528, 529, 530 
[19] Seuren, P. A. M., “A reaction to Jackendoff's discussion note,” Language, vol. 88 (2012), pp. 174-76. 500

[20] Seuren, P. A. M., From Whorf to Montague: Explorations in the Theory of Language, Oxford Univ. Press, Oxford, 2013. 500, 530

[21] Seuren, P. A. M., and D. Jaspers, "Logico-cognitive structure in the lexicon," to appear in Language, (2014). 527, 530

[22] Van Fraassen, B. C., Formal Semantics and Logic, Macmillan, New York, 1971. 529

[23] Zadeh, L. A., "Fuzzy logic and approximate reasoning," Synthese, vol. 30 (1975), pp. 407-28. Zbl 0319.02016. 528

\section{Acknowledgments}

This study owes much to intensive discussions with Dany Jaspers, of HogeschoolUniversiteit Brussel, in the spring of 2012. I am also indebted to Hans Smessaert of Katholieke Universiteit Leuven for useful comments regarding the disposition of the vertices in the logical diagrams. The anonymous reviewers deserve my thanks for a number of useful and constructive criticisms and suggestions, which have improved this study considerably.

Max Planck Institute for Psycholinguistics

PO Box 310

6500 AH Nijmegen

The Netherlands

pieter.seuren@mpi.nl

http://www.mpi.nl/people/seuren-pieter 